Article

\title{
Bioactive Constituents from the Whole Plants of Gentianella acuta (Michx.) Hulten
}

\author{
Zhijuan Ding ${ }^{1,2}$, Yanxia Liu 1,2, Jingya Ruan ${ }^{1,2}$, Shengcai Yang ${ }^{1,2}$, Haiyang Yu ${ }^{1,2}$, \\ Meiling Chen ${ }^{1,2}$, Yi Zhang ${ }^{1,2, *}$ and Tao Wang ${ }^{1,2, *}$ \\ 1 Tianjin State Key Laboratory of Modern Chinese Medicine, 312 Anshanxi Road, Nankai District, \\ Tianjin 300193, China; 15222792071@163.com (Z.D.); liuyanxia210@163.com (Y.L.); \\ Ruanjy19930919@163.com (J.R.); 15122473723@163.com (S.Y.); yuhaiyang19830116@hotmail.com (H.Y.); \\ custard.chen@tjutcm.edu.cn (M.C.) \\ 2 Tianjin Key Laboratory of TCM Chemistry and Analysis, Institute of Traditional Chinese Medicine, \\ Tianjin University of Traditional Chinese Medicine, 312 Anshanxi Road, Nankai District, \\ Tianjin 300193, China \\ * Correspondence: zhwwxzh@263.net (Y.Z.); wangtao@tjutcm.edu.cn (T.W.); \\ Tel./Fax: +86-22-5959-6163 (Y.Z. \& T.W.)
}

Received: 6 July 2017; Accepted: 4 August 2017; Published: 6 August 2017

\begin{abstract}
As a Mongolian native medicine and Ewenki folk medicinal plant, Gentianella acuta has been widely used for the treatment of diarrhea, hepatitis, arrhythmia, and coronary heart disease. In the course of investigating efficacy compounds to treat diarrhea using a mouse isolated intestine tissue model, we found $70 \% \mathrm{EtOH}$ extract of G. acuta whole plants had an inhibitory effect on intestine contraction tension. Here, nineteen constituents, including five new compounds, named as gentiiridosides A (1), B (2), gentilignanoside A (3), (1R)-2,2,3-trimethyl-4hydroxymethylcyclopent-3-ene-1-methyl-O- $\beta$-D-glucopyranoside (4), and (3Z)-3-hexene- 1,5-diol $1-O-\alpha$-L-arabinopyranosyl $(1 \rightarrow 6)-\beta$-D-glucopyranoside (5) were obtained from it. The structures of them were elucidated by chemical and spectroscopic methods. Furthermore, the inhibitory effects on motility of mouse isolated intestine tissue of the above mentioned compounds and other thirteen iridoid- and secoiridoid-type monoterpenes $(7-10,13-16,18,19,21,22$, and 25) previously obtained in the plant were analyzed. As results, new compound 5, some secoiridoid-type monoterpenes 7, 10, 12-14, 16, and 17, as well as 7-O-9'-type lignans 31 and 32 displayed significant inhibitory effect on contraction tension at $40 \mu \mathrm{M}$.
\end{abstract}

Keywords: Gentianella acuta (Michx.) Hulten; lignan; iridoid- and secoiridoid-type monoterpene; intestine motility; mouse isolated intestine tissue

\section{Introduction}

Gentianella acuta (Michx.) Hulten belongs to the family Gentianaceae, distributed mainly in the north of China, Mongolia plateau, Siberia, and Far East areas of Russia [1]. As a Mongolian native medicine and Ewenki folk medicinal plant, G. acuta has been widely used for the treatment of diarrhea, hepatitis, arrhythmia, coronary heart disease, jaundice, fever, and headache [1-3]. In the course of investigating efficacy compounds to treat diarrhea using a mouse isolated intestine tissue model, we found that a $70 \% \mathrm{EtOH}$ extract of G. acuta whole plants had an inhibitory effect on intestine contraction tension. Moreover, eighteen xanthones had been obtained and their inhibitory effects on the model were assayed. As results, some xanthones were found to have a significant reducing effect on intestine contraction tension [4]. Additionally, for xanthones, monoterpenes, and lignans were elucidated to be main constituents in the plant in our continuing study, among them, the spectroscopy data of thirteen iridoid- and secoiridoid-type monoterpenes had been reported $[5,6]$ by us. Here, 
we obtained other nineteen constituents, including five new compounds (1-5) (Figure 1) and fourteen known ones $(\mathbf{6}, \mathbf{1 1}, \mathbf{1 2}, \mathbf{1 7}, \mathbf{2 0}, \mathbf{2 3}, \mathbf{2 4}, \mathbf{2 6}-\mathbf{3 2}$ ) (Figure 2). Do they have an inhibitory effect on motility of mouse isolated intestine tissue, too? In this paper, we describe the isolation and structure elucidation of them, along with evaluations of their inhibitory effects on intestine contraction tension.
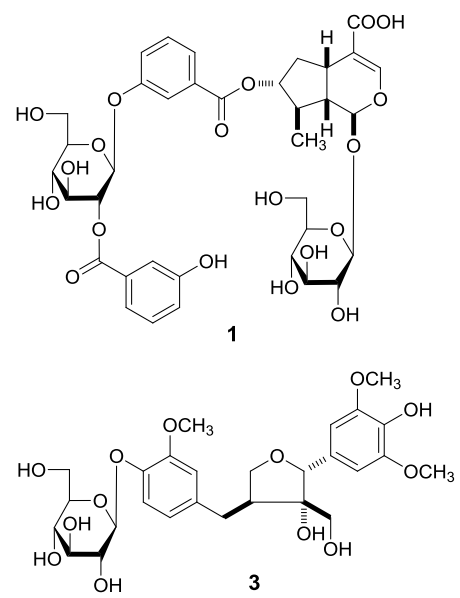
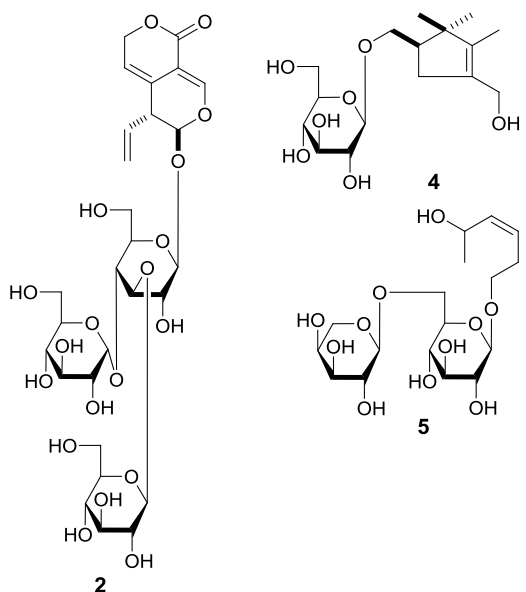

Figure 1. The new compounds 1-5 obtained from the whole plant of G. acuta.

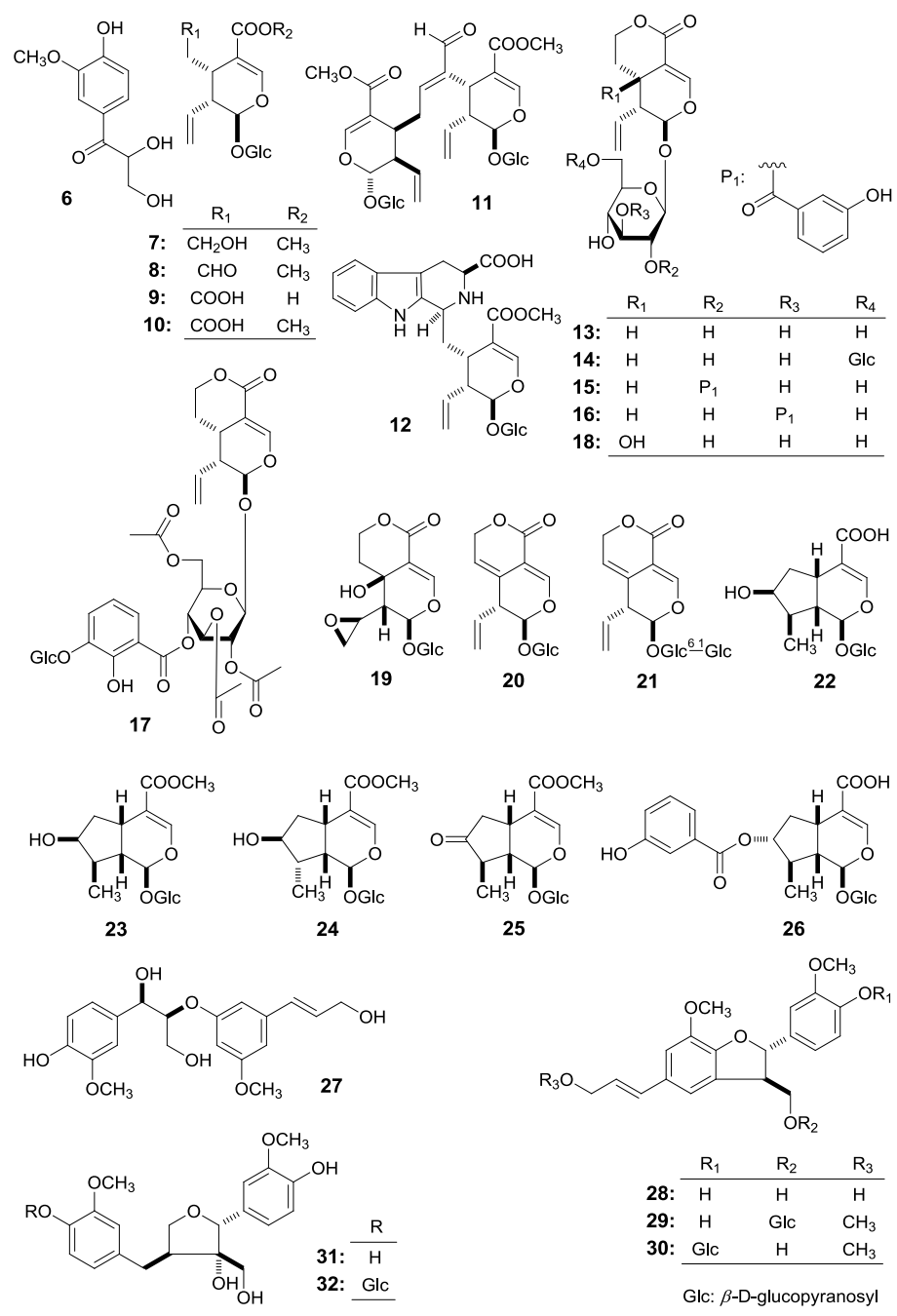

Figure 2. The known compounds (6-32) obtained from the whole plant of G. acuta. 


\section{Results and Discussion}

During the course of our continuous studies on bioactive constituents from a 95\% EtOH eluate of $\mathrm{D} 101 \mathrm{CC}$ and $\mathrm{CHCl}_{3}$ layer [4-6], obtained from the whole plants of G. acuta, nineteen constituents, including five new compounds, named as gentiiridosides A (2), B (2), gentilignanoside A (3), (1R)-2,2,3-trimethyl-4-hydroxymethylcyclopent-3-ene-1-methyl-O- $\beta$-D-glucopyranoside (4), and (3Z)-3-hexene-1,5-diol 1-O- $\alpha$-L-arabinopyranosyl $(1 \rightarrow 6)-\beta$-D-glucopyranoside (5) (Figure 1 ), together with fourteen known ones, 2,3-dihydroxy-1-(4-hydroxy-3-methoxyphenyl)-propan-1-one (6) [7] (E)-aldosecologanin (11) [8], 5 $\alpha$-carboxystrictosidine (12) [9], trifloroside (17) [10], gentiopicroside (20) [11], loganin (23) [12], 8-epiloganin (24) [13], swertiaside (26) [14], (7R,8S)-erythro-4,7,9,9'tetrahydroxy-3,3'-dimethoxy-8-O-4'-neolignan (27) [15,16], (7S,8R)-dehydrodiconiferyl alcohol (28) [17-19], plucheoside $\mathrm{D}_{3}$ (29) $[17,18,20],(7 S, 8 R)-9^{\prime}$-methoxy-dehydrodiconiferyl alcohol 4-O- $\beta$ D-glucopyranoside (30) [17,18,21], (-)-berchemol (31) [22,23], and berchemol-4'-O- $\beta$-D-glucoside (32) [22,24] (Figure 2), were further obtained. Among the known isolates, 6, 11, 12, 17, 23, and 27-32 were isolated from the genus firstly.

This paper will elucidate the isolation and structure of the new compounds. Meanwhile, the effects of the abovementioned compounds and previously-isolated thirteen iridoid- and secoiridoid-type monoterpenes, secologanol (7) [5], secologanin [6] (8), secologanoside (9) [5], secoxyloganin (10) [5], sweroside (13) [6], swertiapunimarin (14) [5], deacetylcentapicrin (15) [6], decentapicrin A (16) [6], swertiamarin (18) [5], eustomoside (19) [5], 6'-O- $\beta$-D-glucopyranosyl gentiopicroside (21) [5], loganic acid (22) [6], 7-ketologanin (25) [6] (Figure 2) on the motility of mouse isolated intestine tissue were determined.

Gentiiridoside $A(\mathbf{1})$ was isolated as a white powder with negative optical rotation $\left[[\alpha]_{\mathrm{D}}^{25}\right.$ $-90.0^{\circ}$ ( c 0.14, MeOH)]. Negative high resolution electrospray ionization-time of flight-mass spectra (HRESI-TOF-MS) afforded [M - H] ${ }^{-}$at $m / z 777.2261$ (calcd for $\mathrm{C}_{36} \mathrm{H}_{41} \mathrm{O}_{19}, 777.2248$ ), supporting a molecular formula of $\mathrm{C}_{36} \mathrm{H}_{42} \mathrm{O}_{19}$ for 1 . The absorption bands showed in the infrared (IR) spectrum suggested the presence of hydroxyl $\left(3372 \mathrm{~cm}^{-1}\right), \alpha, \beta$-unsaturated carbonyl $\left(1712 \mathrm{~cm}^{-1}\right)$, aromatic ring $\left(1635,1588,1486 \mathrm{~cm}^{-1}\right)$, and $O$-glycosidic linkage $\left(1078 \mathrm{~cm}^{-1}\right)$. The sugars in 1 were found to be D-glucose by acid hydrolysis with $1 \mathrm{M} \mathrm{HCl}$ [4]. The ${ }^{1} \mathrm{H},{ }^{13} \mathrm{C}$-nuclear magnetic resonance (NMR) spectra (Table 1) and various two-dimensional (2D) NMR spectra, including ${ }^{1} \mathrm{H}^{1} \mathrm{H}$ chemical-shift correlation spectroscopy $\left({ }^{1} \mathrm{H}^{1} \mathrm{H}\right.$ COSY), heteronuclear single quantum correlation (HSQC), heteronuclear multiple bond correlation (HMBC) displayed signals assignable to two 3-hydroxy benzoyl $\{[\delta 7.42(1 \mathrm{H}, \mathrm{br}$. $\mathrm{d}, \mathrm{ca}$. $\left.J=8 \mathrm{~Hz}, \mathrm{H}-4^{\prime \prime}\right), 7.48\left(1 \mathrm{H}, \mathrm{dd}, J=7.5,7.5 \mathrm{~Hz}, \mathrm{H}-5^{\prime \prime}\right), 7.84\left(1 \mathrm{H}\right.$, br. d, ca. $\left.J=8 \mathrm{~Hz}, \mathrm{H}-6^{\prime \prime}\right), 7.89(1 \mathrm{H}$, br. s, $\left.\left.\mathrm{H}-2^{\prime \prime}\right) ; \delta_{\mathrm{C}} 166.1\left(\mathrm{C}-7^{\prime \prime}\right)\right]$; [ $\delta 7.45\left(1 \mathrm{H}\right.$, br. d, ca. $\left.J=8 \mathrm{~Hz}, \mathrm{H}-4^{\prime \prime \prime \prime}\right), 7.54\left(1 \mathrm{H}, \mathrm{dd}, J=7.5,7.5 \mathrm{~Hz}, \mathrm{H}-5^{\prime \prime \prime \prime}\right), 7.82$ $\left(1 \mathrm{H}\right.$, br. s, H-2 $\left.{ }^{\prime \prime \prime \prime}\right), 7.88\left(1 \mathrm{H}\right.$, br. $\left.\left.\left.\mathrm{d}, \mathrm{ca} . J=8 \mathrm{~Hz}, \mathrm{H}-6^{\prime \prime \prime \prime}\right) ; \delta_{\mathrm{C}} 166.8\left(\mathrm{C}-7^{\prime \prime \prime \prime}\right)\right]\right\}$, and two $\beta$-D-glucopyranosyl [ $\left.\delta 4.69\left(1 \mathrm{H}, \mathrm{d}, J=7.5 \mathrm{~Hz}, \mathrm{H}-1^{\prime}\right), 5.02\left(1 \mathrm{H}, \mathrm{d}, J=8.0 \mathrm{~Hz}, \mathrm{H}-1^{\prime \prime \prime}\right)\right]$. On the other hand, thirty-six carbon signals were shown in its ${ }^{13} \mathrm{C}$-NMR spectrum, in addition to twenty-six carbon signals occupied by the abovementioned fragments, the other 10 carbon signals, together with the relative proton signals [ $\delta 5.50$ $(1 \mathrm{H}, \mathrm{d}, J=3.0 \mathrm{~Hz}, \mathrm{H}-1), 7.46(1 \mathrm{H}, \mathrm{s}, \mathrm{H}-3)]$ indicated the aglycon of 1 was iridoid. As shown in Figure 3, the ${ }^{1} \mathrm{H}^{1} \mathrm{H}$ COSY experiment on 1 suggested the existence of five partial structures. Furthermore, in the HMBC experiment, long-range correlations from $\delta_{\mathrm{H}} 5.50(\mathrm{H}-1)$ to $\delta_{\mathrm{C}} 152.4(\mathrm{C}-3) ; \delta_{\mathrm{H}} 7.46(\mathrm{H}-3)$ to $\delta_{\mathrm{C}} 32.5(\mathrm{C}-5), 96.2(\mathrm{C}-1), 112.8(\mathrm{C}-4), 170.9(\mathrm{C}-11) ; \delta_{\mathrm{H}} 4.96(\mathrm{H}-7)$ to $\delta_{\mathrm{C}} 166.1\left(\mathrm{C}-7^{\prime \prime}\right) ; \delta_{\mathrm{H}} 4.69\left(\mathrm{H}-1^{\prime}\right)$ to $\delta_{\mathrm{C}} 96.2(\mathrm{C}-1) ; \delta_{\mathrm{H}} 7.89\left(\mathrm{H}-2^{\prime \prime}\right), 7.84\left(\mathrm{H}-6^{\prime \prime}\right)$ to $\delta_{\mathrm{C}} 166.1\left(\mathrm{C}-7^{\prime \prime}\right) ; \delta_{\mathrm{H}} 5.02\left(\mathrm{H}-1^{\prime \prime \prime}\right)$ to $\delta_{\mathrm{C}} 159.2\left(\mathrm{C}-3^{\prime \prime}\right) ; \delta_{\mathrm{H}}$ $3.51\left(\mathrm{H}-2^{\prime \prime \prime}\right)$ to $\delta_{\mathrm{C}} 166.8\left(\mathrm{C}-7^{\prime \prime \prime \prime}\right) ; \delta_{\mathrm{H}} 7.82\left(\mathrm{H}-2^{\prime \prime \prime \prime}\right), 7.88\left(\mathrm{H}-6^{\prime \prime \prime \prime}\right)$ to $\delta_{\mathrm{C}} 166.8\left(\mathrm{C}-7^{\prime \prime \prime \prime}\right)$ were observed. Therefore, the planar structure of $\mathbf{1}$ was constructed. The relative configuration of $\mathbf{1}$ was determined by a nuclear Overhauser effect spectroscopy (NOESY) experiment, and NOE correlations were observed between $\mathrm{H}-1$ and $\mathrm{H}-8$; $\mathrm{H}-5$ and $\mathrm{H}-7 ; \mathrm{H}_{3}-10$ and $\mathrm{H}-7, \mathrm{H}-9$. The ${ }^{1} \mathrm{H}$ and ${ }^{13} \mathrm{C}-\mathrm{NMR}$ sepctra of $\mathbf{1}$ were found very similar to those of swertiaside (26) [14], except that a 2-(3-hydroxybenzoyl)- $\beta$-D-glucopyranosyl appeared at the $3^{\prime \prime}$-position in $\mathbf{1}$. Consequently, the structure of gentiiridoside A (1) was determined. 
Table $1 .{ }^{1} \mathrm{H}$ and ${ }^{13} \mathrm{C}-\mathrm{NMR}$ data for $\mathbf{1}$ in $\mathrm{CD}_{3} \mathrm{OD}$.

\begin{tabular}{|c|c|c|c|c|c|}
\hline No. & $\delta_{C}$ & $\delta_{H}(J$ in $\mathbf{H z})$ & No. & $\delta_{C}$ & $\delta_{H}(J$ in $\mathbf{H z})$ \\
\hline 1 & 96.2 & $5.50(\mathrm{~d}, 3.0)$ & $3^{\prime \prime}$ & 159.2 & - \\
\hline 3 & 152.4 & $7.46(\mathrm{~s})$ & $4^{\prime \prime}$ & 123.5 & 7.42 (br. d, ca. 8) \\
\hline 4 & 112.8 & - & $5^{\prime \prime}$ & 131.0 & $7.48(\mathrm{dd}, 7.5,7.5)$ \\
\hline 5 & 32.5 & 3.05 (q like, $c a .7)$ & $6^{\prime \prime}$ & 125.1 & 7.84 (br. d, ca. 8) \\
\hline \multirow[t]{2}{*}{6} & 38.0 & $2.00(\mathrm{~m})$ & $7^{\prime \prime}$ & 166.1 & - \\
\hline & & $2.55(\mathrm{ddd}, 6.5,7.0,13.5)$ & $1^{\prime \prime \prime}$ & 102.3 & $5.02(\mathrm{~d}, 8.0)$ \\
\hline 7 & 83.7 & $4.96(\mathrm{ddd}, 4.5,7.0,10.5)$ & $2^{\prime \prime \prime}$ & 77.9 & 3.51 (m, overlapped) \\
\hline 8 & 43.0 & $2.12(\mathrm{~m})$ & $3^{\prime \prime \prime}$ & 74.8 & 3.53 (m, overlapped) \\
\hline 9 & 48.7 & $2.00(\mathrm{~m}$, overlapped $)$ & $4^{\prime \prime \prime}$ & 71.4 & 3.32 (m, overlapped) \\
\hline 10 & 18.4 & $1.23(\mathrm{~d}, 7.0)$ & $5^{\prime \prime \prime}$ & 78.1 & 3.33 (m, overlapped) \\
\hline 11 & 170.9 & - & $6^{\prime \prime \prime}$ & 62.6 & $3.66(\mathrm{dd}, 4.0,11.5)$ \\
\hline $1^{\prime}$ & 100.2 & $4.69(\mathrm{~d}, 7.5)$ & & & 3.84 (br. d, ca. 12) \\
\hline $2^{\prime}$ & 74.6 & $3.22(\mathrm{dd}, 7.5,8.5)$ & $1^{\prime \prime \prime \prime \prime}$ & 133.1 & - \\
\hline $3^{\prime}$ & 77.8 & $3.40(\mathrm{dd}, 8.5,8.5)$ & $2^{\prime \prime \prime \prime \prime}$ & 123.9 & 7.82 (br. s) \\
\hline $4^{\prime}$ & 71.3 & $3.46(\mathrm{dd}, 8.5,8.5)$ & $3^{\prime \prime \prime \prime \prime}$ & 152.3 & - \\
\hline $5^{\prime}$ & 78.2 & $3.51(\mathrm{~m}$, overlapped $)$ & $4^{\prime \prime \prime \prime \prime}$ & 127.7 & 7.45 (br. d, ca. 8) \\
\hline \multirow[t]{2}{*}{$6^{\prime}$} & 62.4 & $3.75(\mathrm{dd}, 5.0,11.5)$ & $5^{\prime \prime \prime \prime}$ & 130.8 & $7.54(\mathrm{dd}, 7.5,7.5)$ \\
\hline & & 3.92 (br. d, ca. 12) & $6^{\prime \prime \prime \prime \prime}$ & 128.1 & 7.88 (br. d, ca. 8) \\
\hline $1^{\prime \prime}$ & 131.7 & - & $7^{\prime \prime \prime \prime \prime}$ & 166.8 & - \\
\hline $2^{\prime \prime}$ & 119.3 & 7.89 (br. s) & & & \\
\hline
\end{tabular}
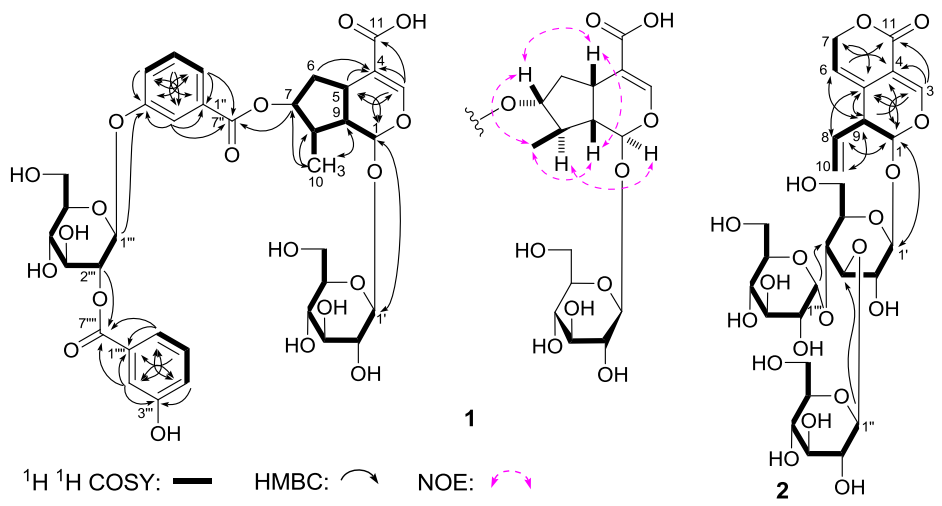

Figure 3. The main ${ }^{1} \mathrm{H}^{1} \mathrm{H}$ COSY, HMBC correlations of $\mathbf{1}$ and $\mathbf{2}$, and NOE correlations of $\mathbf{1}$.

Gentiiridoside B (2) was obtained as a white powder with negative optical rotation $\left[[\alpha]_{\mathrm{D}}^{25}-36.7^{\circ}\right.$ (c 0.12, MeOH)]. HRESI-TOF-MS exhibited a molecular ion peak at $m / z 679.1023[\mathrm{M}-\mathrm{H}]^{-}$, and revealed the molecular formula $\mathrm{C}_{28} \mathrm{H}_{40} \mathrm{O}_{18}$ (calcd for $\mathrm{C}_{28} \mathrm{H}_{39} \mathrm{O}_{18}, 679.1033$ ) for it. Acid hydrolysis of 2 with $1 \mathrm{M} \mathrm{HCl}$ afforded D-glucose, whose absolute configuration was determined by HPLC analysis [4]. The ${ }^{1} \mathrm{H}$ and ${ }^{13} \mathrm{C}$-NMR (Table 2) spectra of 2 indicated the presence of two $\beta$-D-glucopyranosyl [ $\delta 4.27$ $\left.\left(1 \mathrm{H}, \mathrm{d}, J=7.5 \mathrm{~Hz}, \mathrm{H}-1^{\prime \prime}\right), 4.49\left(1 \mathrm{H}, \mathrm{d}, J=8.0 \mathrm{~Hz}, \mathrm{H}-1^{\prime}\right)\right]$, and one $\alpha$-D-glucopyranosyl [ $\delta 4.91(1 \mathrm{H}, \mathrm{d}$, $\left.\left.J=3.5 \mathrm{~Hz}, \mathrm{H}-\mathrm{-}^{\prime \prime \prime}\right)\right]$. Twenty-eight carbon signals were displayed in its ${ }^{13} \mathrm{C}-\mathrm{NMR}$ spectrum, except for the above mentiond moieties, the other ten signals as well as their relative ${ }^{1} \mathrm{H}$-NMR signals $[\delta 5.21(2 \mathrm{H}$, $\left.\left.\mathrm{m}, \mathrm{H}_{2}-10\right), 5.59(1 \mathrm{H}, \mathrm{d}, J=3.0 \mathrm{~Hz}, \mathrm{H}-1), 5.72(1 \mathrm{H}, \mathrm{ddd}, J=6.5,10.5,17.5 \mathrm{~Hz}, \mathrm{H}-8), 7.41(1 \mathrm{H}, \mathrm{s}, \mathrm{H}-3)\right]$ suggested the aglycon of 2 was the same as that of gentiopicroside (20) [11]. Finally, the long-range correlations from $\delta_{\mathrm{H}} 5.59(\mathrm{H}-1)$ to $\delta_{\mathrm{C}} 124.9(\mathrm{C}-5), 148.8(\mathrm{C}-3) ; \delta_{\mathrm{H}} 7.41(\mathrm{H}-3)$ to $\delta_{\mathrm{C}} 96.4(\mathrm{C}-1), 103.2(\mathrm{C}-4)$, $124.9(\mathrm{C}-5), 162.7(\mathrm{C}-11) ; \delta_{\mathrm{H}} 5.65(\mathrm{H}-6)$ to $\delta_{\mathrm{C}} 44.3(\mathrm{C}-9), 103.2(\mathrm{C}-4) ; \delta_{\mathrm{H}} 4.97,5.04\left(\mathrm{H}_{2}-7\right)$ to $\delta_{\mathrm{C}} 124.9(\mathrm{C}-5)$, $162.7(\mathrm{C}-11) ; \delta_{\mathrm{H}} 5.72(\mathrm{H}-8)$ to $\delta_{\mathrm{C}} 96.4(\mathrm{C}-1), 124.9(\mathrm{C}-5) ; \delta_{\mathrm{H}} 3.31$ (H-9) to $\delta_{\mathrm{C}} 103.2(\mathrm{C}-4), 116.1(\mathrm{C}-6), 124.9$ $(\mathrm{C}-5) ; \delta_{\mathrm{H}} 4.49\left(\mathrm{H}-1^{\prime}\right)$ to $\delta_{\mathrm{C}} 96.4(\mathrm{C}-1) ; \delta_{\mathrm{H}} 4.27\left(\mathrm{H}-1^{\prime \prime}\right)$ to $\delta_{\mathrm{C}} 76.7\left(\mathrm{C}-1^{\prime}\right) ; \delta_{\mathrm{H}} 4.91\left(\mathrm{H}-1^{\prime \prime \prime}\right)$ to $\delta_{\mathrm{C}} 69.9\left(\mathrm{C}-1^{\prime \prime}\right)$ were observed in the HMBC spectrum. Then, the structure of gentiiridoside B (2) was clarified. 
Table 2. ${ }^{1} \mathrm{H}$ and ${ }^{13} \mathrm{C}-\mathrm{NMR}$ data for 2 in DMSO- $d_{6}$.

\begin{tabular}{|c|c|c|c|c|c|}
\hline No. & $\delta_{\mathrm{C}}$ & $\delta_{\mathrm{H}}(J$ in $\mathrm{Hz})$ & No. & $\delta_{\mathrm{C}}$ & $\delta_{\mathrm{H}}(J$ in $\mathrm{Hz})$ \\
\hline 1 & 96.4 & $5.59(\mathrm{~d}, 3.0)$ & $6^{\prime}$ & 61.0 & $3.44(\mathrm{~m}$, overlapped $)$ \\
\hline 3 & 148.8 & $7.41(\mathrm{~s})$ & & & 3.68 (br. d, ca. 12) \\
\hline 4 & 103.2 & - & $1^{\prime \prime}$ & 96.8 & $4.27(\mathrm{~d}, 7.5)$ \\
\hline 5 & 124.9 & - & $2^{\prime \prime}$ & 74.7 & $2.90(\mathrm{dd}, 7.5,8.5)$ \\
\hline 6 & 116.1 & $5.65(\mathrm{~m})$ & $3^{\prime \prime}$ & 76.6 & $3.12(\mathrm{~m}$, overlapped $)$ \\
\hline \multirow[t]{2}{*}{7} & 69.1 & $4.97(\mathrm{dd}, 3.0,18.0)$ & $4^{\prime \prime}$ & 70.2 & 3.05 (m, overlapped) \\
\hline & & 5.04 (br. d, ca. 18) & $5^{\prime \prime}$ & 76.7 & 3.03 (m, overlapped) \\
\hline 8 & 134.0 & $5.72(\mathrm{ddd}, 6.5,10.5,17.5)$ & $6^{\prime \prime}$ & 61.1 & $3.44(\mathrm{~m}$, overlapped $)$ \\
\hline 9 & 44.3 & $3.31(\mathrm{~m})$ & & & 3.66 (br. d, ca. 12) \\
\hline 10 & 117.9 & $5.21(\mathrm{~m})$ & $1^{\prime \prime \prime}$ & 92.1 & $4.91(\mathrm{~d}, 3.5)$ \\
\hline 11 & 162.7 & - & $2^{\prime \prime \prime}$ & 72.3 & $3.12(\mathrm{~m}$, overlapped $)$ \\
\hline $1^{\prime}$ & 98.7 & $4.49(\mathrm{~d}, 8.0)$ & $3^{\prime \prime \prime}$ & 72.7 & $3.42(\mathrm{~m}$, overlapped $)$ \\
\hline $2^{\prime}$ & 73.0 & $2.95(\mathrm{dd}, 8.0,8.5)$ & $4^{\prime \prime \prime}$ & 70.5 & 3.05 (m, overlapped) \\
\hline $3^{\prime}$ & 76.7 & 3.15 (m, overlapped) & $5^{\prime \prime \prime}$ & 71.9 & $3.57(\mathrm{~m})$ \\
\hline $4^{\prime}$ & 69.9 & 3.03 (m, overlapped) & $6^{\prime \prime \prime}$ & 61.1 & $3.44(\mathrm{~m}$, overlapped $)$ \\
\hline $5^{\prime}$ & 77.3 & 3.15 (m, overlapped) & & & \\
\hline
\end{tabular}

Gentilignanoside A (3) was obtained as a white powder that exhibited negative optical rotation $\left[[\alpha]_{\mathrm{D}}^{25}-36.0^{\circ}\right.$ (c 0.10, MeOH)]. The molecular formula, $\mathrm{C}_{27} \mathrm{H}_{34} \mathrm{O}_{13}$, of 3 was determined from Q-TOF-ESI-MS analysis $\left(m / z 567.2083[\mathrm{M}-\mathrm{H}]^{-}\right.$, calcd for $\left.\mathrm{C}_{27} \mathrm{H}_{33} \mathrm{O}_{13}, 567.2083\right)$. Its IR spectrum showed absorption bands due to hydroxyl $\left(3368 \mathrm{~cm}^{-1}\right)$, aromatic ring $\left(1613,1513,1463 \mathrm{~cm}^{-1}\right)$, and O-glycosidic linkage $\left(1073 \mathrm{~cm}^{-1}\right)$. The ${ }^{1} \mathrm{H},{ }^{13} \mathrm{C}-\mathrm{NMR}$ spectra (Table 3 ) and kinds of 2D NMR spectra $\left({ }^{1} \mathrm{H}^{1} \mathrm{H}\right.$ COSY, HSQC, HMBC) showed signals ascribable to one ABX-type aromatic protons $[\delta 6.77(1 \mathrm{H}$, br. d, ca. $\left.J=8 \mathrm{~Hz}, \mathrm{H}-6^{\prime}\right), 6.90(1 \mathrm{H}$, br. s, H-2' $\left.), 7.10\left(1 \mathrm{H}, \mathrm{d}, J=8.0 \mathrm{~Hz}, \mathrm{H}-5^{\prime}\right)\right]$, one 1,3,4,5-symmetrical substituted phenyl group $[\delta 6.64(2 \mathrm{H}, \mathrm{s}, \mathrm{H}-2,6)]$, two methylene bearing oxygen funtion $\{\delta[3.64,3.80$ (1H each, both $\mathrm{m}$, overlapped, $\left.\mathrm{H}_{2}-9\right)$ ], [3.64 $\left(1 \mathrm{H}, \mathrm{m}\right.$, overlapped), $\left.\left.\left.4.06(1 \mathrm{H}, \mathrm{dd}, \mathrm{J}=7.5,7.5 \mathrm{~Hz}), \mathrm{H}_{2}-9^{\prime}\right]\right]\right\}$, three methoxyl [ $\left.\delta 3.84\left(6 \mathrm{H}, \mathrm{s}, 3,5-\mathrm{OCH}_{3}\right), 3.86\left(3 \mathrm{H}, \mathrm{s}, 3^{\prime}-\mathrm{OCH}_{3}\right)\right]$, and one $\beta$-D-glucopyranosyl [ $\delta 4.88$ $\left.\left(1 \mathrm{H}, \mathrm{d}, J=7.5 \mathrm{~Hz}, \mathrm{H}-1^{\prime \prime}\right)\right]$. The planar structure of 3 was constructed by the assignment of the ${ }^{1} \mathrm{H}$ ${ }^{1} \mathrm{H}$ COSY and $\mathrm{HMBC}$ experiments as shown in Figure 4. The ${ }^{1} \mathrm{H}^{1} \mathrm{H}$ COSY experiment indicated the presence of three partial moieties. On the other hand, in the HMBC experiment, long-range correlations were found from the following proton and carbon pairs: $\delta_{\mathrm{H}} 6.64(\mathrm{H}-2,6)$ to $\delta_{\mathrm{C}} 129.9(\mathrm{C}-1), 136.2(\mathrm{C}-4)$, $148.9(\mathrm{C}-3,5) ; \delta_{\mathrm{H}} 4.84(\mathrm{H}-7)$ to $\delta_{\mathrm{C}} 51.8\left(\mathrm{C}-8^{\prime}\right), 64.6(\mathrm{C}-9), 106.2(\mathrm{C}-2,6), 129.9(\mathrm{C}-1) ; \delta_{\mathrm{H}} 3.64,3.80\left(\mathrm{H}_{2}-9\right)$ to $\delta_{\mathrm{C}} 51.8\left(\mathrm{C}-8^{\prime}\right), 83.3(\mathrm{C}-8), 85.8(\mathrm{C}-7) ; \delta_{\mathrm{H}} 6.90\left(\mathrm{H}-2^{\prime}\right)$ to $\delta_{\mathrm{C}} 35.1\left(\mathrm{C}-7^{\prime}\right), 122.4\left(\mathrm{C}-6^{\prime}\right), 146.5\left(\mathrm{C}-4^{\prime}\right), 150.9$ $\left(\mathrm{C}-3^{\prime}\right) ; \delta_{\mathrm{H}} 7.10\left({\left.\mathrm{H}-5^{\prime}\right)}^{\prime}\right.$ to $\delta_{\mathrm{C}} 136.9\left(\mathrm{C}-1^{\prime}\right), 146.5\left(\mathrm{C}-4^{\prime}\right), 150.9\left(\mathrm{C}-3^{\prime}\right) ; \delta_{\mathrm{H}} 6.77\left(\mathrm{H}-6^{\prime}\right)$ to $\delta_{\mathrm{C}} 35.1\left(\mathrm{C}-7^{\prime}\right), 114.4$ $\left(\mathrm{C}-2^{\prime}\right), 146.5\left(\mathrm{C}-4^{\prime}\right) ; \delta_{\mathrm{H}} 2.54,3.13\left(\mathrm{H}_{2}-7^{\prime}\right)$ to $\delta_{\mathrm{C}} 72.0\left(\mathrm{C}-9^{\prime}\right), 114.4\left(\mathrm{C}-2^{\prime}\right), 122.4\left(\mathrm{C}-6^{\prime}\right), 136.9\left(\mathrm{C}-1^{\prime}\right) ; \delta_{\mathrm{H}} 2.59$ $\left(\mathrm{H}-8^{\prime}\right)$ to $\delta_{\mathrm{C}} 136.9\left(\mathrm{C}-1^{\prime}\right) ; \delta_{\mathrm{H}} 3.64,4.06\left(\mathrm{H}_{2}-9^{\prime}\right)$ to $\delta_{\mathrm{C}} 35.1\left(\mathrm{C}-7^{\prime}\right), 83.3(\mathrm{C}-8), 85.8(\mathrm{C}-7) ; \delta_{\mathrm{H}} 3.84\left(3,5-\mathrm{OCH}_{3}\right)$ to $\delta_{\mathrm{C}} 148.9(\mathrm{C}-3,5) ; \delta_{\mathrm{H}} 3.86\left(3^{\prime}-\mathrm{OCH}_{3}\right)$ to $\delta_{\mathrm{C}} 150.9\left(\mathrm{C}-3^{\prime}\right) ; \delta_{\mathrm{H}} 4.88\left(\mathrm{H}-1^{\prime \prime}\right)$ to $\delta_{\mathrm{C}} 146.5\left(\mathrm{C}-4^{\prime}\right)$. Furthermore, the relative configuration of 3 was determined by the NOE correlations between $\delta_{\mathrm{H}} 4.84(\mathrm{H}-7)$ and $\delta_{\mathrm{H}}$ $3.64,3.80\left(\mathrm{H}_{2}-9\right) ; \delta_{\mathrm{H}} 3.64,3.80\left(\mathrm{H}_{2}-9\right)$ and $\delta_{\mathrm{H}} 2.54,3.13\left(\mathrm{H}_{2}-7^{\prime}\right)$ observed in its NOESY spectrum. Finally, 3 showed negative Cotton effect at 278 and $232 \mathrm{~nm}$, which indicated the absolute configuration of it was $7 R, 8 S, 8^{\prime} S[22]$.
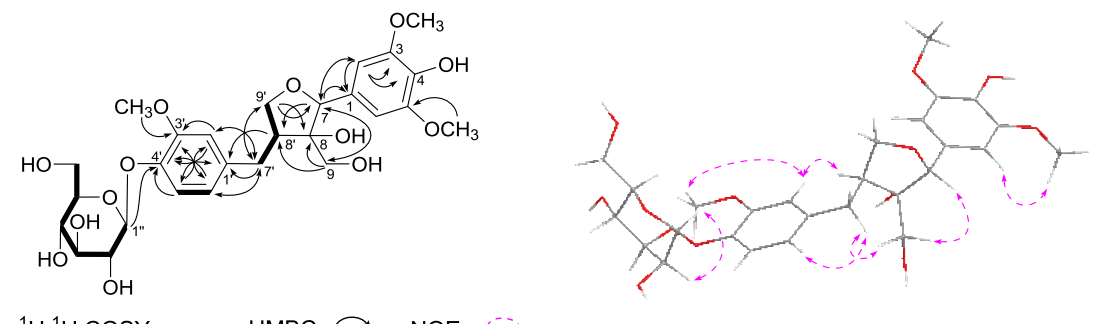

${ }^{1} \mathrm{H}^{1} \mathrm{HCOSY}:-\mathrm{HMBC}: \frown \mathrm{NOE}: r^{-\cdots}$

Figure 4. The main ${ }^{1} \mathrm{H}^{1} \mathrm{H}$ COSY, HMBC, and NOE correlations of 3. 
Table $3 .{ }^{1} \mathrm{H}$ and ${ }^{13} \mathrm{C}-\mathrm{NMR}$ data for 3 in $\mathrm{CD}_{3} \mathrm{OD}$.

\begin{tabular}{|c|c|c|c|c|c|}
\hline No. & $\delta_{C}$ & $\delta_{\mathrm{H}}(J$ in $\mathrm{Hz})$ & No. & $\delta_{C}$ & $\delta_{\mathrm{H}}(J$ in $\mathrm{Hz})$ \\
\hline 1 & 129.9 & - & $7^{\prime}$ & 35.1 & $2.54(\mathrm{dd}, 12.5,12.5)$ \\
\hline 2,6 & 106.2 & $6.64(\mathrm{~s})$ & & & 3.13 (br. d, ca. 13) \\
\hline 3,5 & 148.9 & - & $8^{\prime}$ & 51.8 & $2.59(\mathrm{~m})$ \\
\hline 4 & 136.2 & - & $9^{\prime}$ & 72.0 & 3.64 (m, overlapped) \\
\hline 7 & 85.8 & $4.84(\mathrm{~s})$ & & & $4.06(\mathrm{dd}, 7.5,7.5)$ \\
\hline 8 & 83.3 & - & $3,5-\mathrm{OCH}_{3}$ & 56.8 & $3.84(\mathrm{~s})$ \\
\hline \multirow[t]{2}{*}{9} & 64.6 & $3.64(\mathrm{~m}$, overlapped $)$ & $3^{\prime}-\mathrm{OCH}_{3}$ & 56.8 & $3.86(\mathrm{~s})$ \\
\hline & & $3.80(\mathrm{~m}$, overlapped $)$ & $1^{\prime \prime}$ & 103.1 & $4.88(\mathrm{~d}, 7.5)$ \\
\hline $1^{\prime}$ & 136.9 & - & $2^{\prime \prime}$ & 75.0 & $3.49(\mathrm{dd}, 7.5,8.5)$ \\
\hline $2^{\prime}$ & 114.4 & 6.90 (br. s) & $3^{\prime \prime}$ & 77.9 & $3.45(\mathrm{~m})$ \\
\hline $3^{\prime}$ & 150.9 & - & $4^{\prime \prime}$ & 71.4 & $3.40(\mathrm{~m}$, overlapped $)$ \\
\hline $4^{\prime}$ & 146.5 & - & $5^{\prime \prime}$ & 78.2 & 3.40 (m, overlapped) \\
\hline $5^{\prime}$ & 118.3 & $7.10(\mathrm{~d}, 8.0)$ & $6^{\prime \prime}$ & 62.6 & $3.68(\mathrm{dd}, 5.0,11.5)$ \\
\hline $6^{\prime}$ & 122.4 & 6.77 (br. d, ca. 8) & & & $3.89(\mathrm{~m}$, overlapped $)$ \\
\hline
\end{tabular}

(1R)-2,2,3-Trimethyl-4-hydroxymethylcyclopent-3-ene-1-methyl-O- $\beta$-D-glucopyranoside (4), was obtained as a white powder. It had the molecular formula $\mathrm{C}_{16} \mathrm{H}_{28} \mathrm{O}_{7}$, determined by negative-ion HRESI-TOF-MS $(\mathrm{m} / \mathrm{z} 377.1814 \text { [M }+\mathrm{COOH}]^{-}$, calcd for $\left.\mathrm{C}_{17} \mathrm{H}_{29} \mathrm{O}_{9}, 377.1817\right)$. Its IR spectrum showed absorption bands at 3367, 1636, and $1076 \mathrm{~cm}^{-1}$ ascribable to hydroxyl, olefin, and $O$-glycosidic linkages, respectively. It was reated with $1 \mathrm{M} \mathrm{HCl}$ to give D-glucose [4]. The ${ }^{1} \mathrm{H},{ }^{13} \mathrm{C}-\mathrm{NMR}$ (Table 4) spectra showed signals assignable to three methyl $\left[\delta 0.87,1.09,1.56\right.$ ( $3 \mathrm{H}$ each, all s, $\left.\left.2 \beta, 2 \alpha, 3-\mathrm{CH}_{3}\right)\right]$, two methylene with oxygen function $\left\{\left[\delta 3.66(1 \mathrm{H}, \mathrm{m}\right.\right.$, overlapped $\left.), 3.95(1 \mathrm{H}, \mathrm{dd}, J=6.5,11.0 \mathrm{~Hz}), 1-\mathrm{CH}_{2} \mathrm{OH}\right]$, $\left.4.07\left(2 \mathrm{H}, \mathrm{d}, J=9.0 \mathrm{~Hz}, 4-\mathrm{CH}_{2} \mathrm{OH}\right)\right\}$, one $\beta$-D-glucopyranosyl $\left[\delta 4.26\left(1 \mathrm{H}, \mathrm{d}, J=7.5 \mathrm{~Hz}, \mathrm{H}-1^{\prime}\right)\right]$, one methylene $\left[\delta 2.09(1 \mathrm{H}, \mathrm{dd}, J=8.0,9.0 \mathrm{~Hz}), 2.49(1 \mathrm{H}, \mathrm{dd}, J=8.0,8.0 \mathrm{~Hz}), \mathrm{H}_{2}-5\right]$, together with one methine [ $\delta 2.15(1 \mathrm{H}, \mathrm{m}$, overlapped, $\mathrm{H}-1)]$. The ${ }^{1} \mathrm{H}^{1} \mathrm{H}$ COSY experiment suggested the presence of two partial fragments shown in bold lines (Figure 5). Then, the planar structure of 4 was further elucidated by the long-range correlations from $\delta_{\mathrm{H}} 0.87\left(2 \beta-\mathrm{CH}_{3}\right)$ to $\delta_{\mathrm{C}} 27.2\left(2 \alpha-\mathrm{CH}_{3}\right), 49.0(\mathrm{C}-1), 49.3(\mathrm{C}-2), 143.5(\mathrm{C}-3)$; $\delta_{\mathrm{H}} 1.09\left(2 \alpha-\mathrm{CH}_{3}\right)$ to $\delta_{\mathrm{C}} 20.1\left(2 \beta-\mathrm{CH}_{3}\right), 49.0(\mathrm{C}-1), 49.3(\mathrm{C}-2), 143.5(\mathrm{C}-3) ; \delta_{\mathrm{H}} 1.56\left(3-\mathrm{CH}_{3}\right)$ to $\delta_{\mathrm{C}} 49.3(\mathrm{C}-2)$, $133.1(\mathrm{C}-4), 143.5(\mathrm{C}-3) ; \delta_{\mathrm{H}} 4.07\left(4-\mathrm{CH}_{2} \mathrm{OH}\right)$ to $\delta_{\mathrm{C}} 36.6(\mathrm{C}-5), 133.1(\mathrm{C}-4), 143.5(\mathrm{C}-3) ; \delta_{\mathrm{H}} 2.15,2.48\left(\mathrm{H}_{2}-5\right)$ to $\delta_{\mathrm{C}} 49.3(\mathrm{C}-2), 72.1\left(1-\mathrm{CH}_{2} \mathrm{OH}\right), 133.1(\mathrm{C}-4), 143.5(\mathrm{C}-3) ; \delta_{\mathrm{H}} 4.26\left(\mathrm{H}-1^{\prime}\right)$ to $\delta_{\mathrm{C}} 72.1\left(1-\mathrm{CH}_{2} \mathrm{OH}\right)$ observed in the HMBC spectrum. To determine the sterostructure of it, 4 was treated with $\beta$-glucosidase, to give the aglycon, (1R)-2,2,3-trimethyl-4-hydroxymethylcyclopent-3-ene-1-methanol (4a), which was obtained with negative optical rotation $\left([\alpha]_{\mathrm{D}}-6.5^{\circ}, \mathrm{CHCl}_{3}\right)$ and had only one chiral carbon. Using the the same method reported in literatures [25,26], compared optical rotation of $4 \mathbf{a}$ with that of its simialr compound, $(-)-(R)-\gamma$-necrodol $\left([\alpha]_{D}-21.2^{\circ}, \mathrm{CHCl}_{3}\right)$ [27], the absolute configuration of 4 was elucidated to be $1 R$. Finally, the chemical shift of two methyl at the 2-position was determined by NOE correlations displayed in NOESY experiment. On the basis of above mentioned evidences, the structure of 4 was identified as (1R)-2,2,3-trimethyl-4-hydroxymethylcyclopent-3-ene-1-methyl-O- $\beta$-D-glucopyranoside.

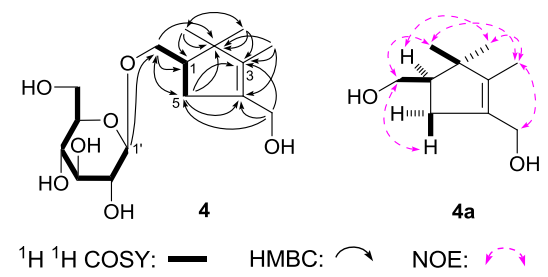

Figure 5. The main ${ }^{1} \mathrm{H}^{1} \mathrm{H}$ COSY, HMBC correlations of $\mathbf{4}$ and $\mathbf{4 a}$. 
Table $4 .{ }^{1} \mathrm{H}$ and ${ }^{13} \mathrm{C}-\mathrm{NMR}$ data for 4 and $4 \mathrm{a}$ in $\mathrm{CD}_{3} \mathrm{OD}$.

\begin{tabular}{|c|c|c|c|c|}
\hline \multirow[b]{2}{*}{ No. } & \multicolumn{2}{|r|}{4} & \multicolumn{2}{|r|}{$4 a$} \\
\hline & $\delta_{\mathrm{C}}$ & $\delta_{\mathrm{H}}(J$ in $\mathrm{Hz})$ & $\delta_{C}$ & $\delta_{\mathrm{H}}(J$ in $\mathrm{Hz})$ \\
\hline 1 & 49.0 & 2.15 (m, overlapped) & 51.7 & $2.00(\mathrm{~m})$ \\
\hline 2 & 49.3 & - & 49.0 & - \\
\hline 3 & 143.5 & - & 143.7 & - \\
\hline 4 & 133.1 & - & 133.2 & - \\
\hline $5 \alpha$ & 36.6 & $2.15(\mathrm{dd}, 8.0,9.0)$ & 36.5 & $2.09(\mathrm{dd}, 8.0,9.0)$ \\
\hline $5 \beta$ & & $2.48(\mathrm{dd}, 8.0,8.0)$ & & $2.49(\mathrm{dd}, 8.0,8.0)$ \\
\hline \multirow[t]{2}{*}{$1-\mathrm{CH}_{2} \mathrm{OH}$} & 72.1 & 3.66 (m, overlapped) & 64.2 & $3.54(\mathrm{dd}, 8.5,11.0)$ \\
\hline & & $3.95(\mathrm{dd}, 6.5,11.0)$ & & $3.72(\mathrm{dd}, 6.5,11.0)$ \\
\hline $2 \alpha-\mathrm{CH}_{3}$ & 27.2 & $1.09(\mathrm{~s})$ & 27.3 & $1.08(\mathrm{~s})$ \\
\hline $2 \beta-\mathrm{CH}_{3}$ & 20.1 & $0.87(\mathrm{~s})$ & 19.9 & $0.85(\mathrm{~s})$ \\
\hline $3-\mathrm{CH}_{3}$ & 9.4 & $1.56(\mathrm{~s})$ & 9.4 & $1.56(\mathrm{~s})$ \\
\hline $4-\mathrm{CH}_{2} \mathrm{OH}$ & 59.4 & $4.07(\mathrm{~m})$ & 59.4 & $\begin{array}{c}4.06,4.10 \text { (both } \mathrm{d} \text {, } \\
12.0 \text { ) }\end{array}$ \\
\hline $1^{\prime}$ & 104.5 & $4.26(\mathrm{~d}, 7.5)$ & & \\
\hline $2^{\prime}$ & 75.2 & $3.17(\mathrm{dd}, 7.5,8.0)$ & & \\
\hline $3^{\prime}$ & 78.3 & $3.34(\mathrm{dd}, 8.0,8.0)$ & & \\
\hline $4^{\prime}$ & 71.7 & 3.29 (m, overlapped) & & \\
\hline $5^{\prime}$ & 78.0 & 3.29 (m, overlapped) & & \\
\hline \multirow[t]{2}{*}{$6^{\prime}$} & 62.8 & $3.66(\mathrm{~m}$, overlapped $)$ & & \\
\hline & & 3.87 (br. d, ca. 12) & & \\
\hline
\end{tabular}

(3Z)-3-Hexene-1,5-diol 1-O- $\alpha$-L-arabinopyranosyl(1 $\rightarrow 6)$ - $\beta$-D-glucopyranoside (5) was obtained as a white powder with negative optical rotation $\left[[\alpha]_{\mathrm{D}}^{25}-14.5^{\circ}(\right.$ c $\left.0.11, \mathrm{MeOH})\right]$. Its molecular formula, $\mathrm{C}_{17} \mathrm{H}_{30} \mathrm{O}_{11}\left(\mathrm{~m} / z 455.1773[\mathrm{M}+\mathrm{COOH}]^{-}\right.$; calcd for $\left.\mathrm{C}_{18} \mathrm{H}_{31} \mathrm{O}_{13}, 455.1770\right)$, was recorded by Q-TOF-ESI-MS. Furthermore, using acid hydrolysis and HPLC analysis, the presence of D-glucose and L-arabinose in 5 was revealed [4]. The ${ }^{1} \mathrm{H},{ }^{13} \mathrm{C}-\mathrm{NMR}$ spectra (Table 5) and 2D NMR $\left({ }^{1} \mathrm{H}{ }^{1} \mathrm{H}\right.$ COSY, HSQC, HMBC) spectra indicated the presence of two olefinic protons $[\delta 5.45,5.47(1 \mathrm{H}$ each, both $\mathrm{m}$, $\mathrm{H}-3$ and 4$)]$, one methoxyl [ $\left.\delta 1.20\left(3 \mathrm{H}, \mathrm{d}, J=6.0 \mathrm{~Hz}, \mathrm{H}_{3}-6\right)\right]$, one $\beta$-D-glucopyranosyl [ $\delta 4.27(1 \mathrm{H}, \mathrm{d}$, $\left.\left.J=7.5 \mathrm{~Hz}, \mathrm{H}-1^{\prime}\right)\right]$, along with one $\alpha$-L-arabinopyranosyl [ $\left.\delta 4.31\left(1 \mathrm{H}, \mathrm{d}, J=6.5 \mathrm{~Hz}, \mathrm{H}-1^{\prime \prime}\right)\right]$. The planar structure of 5 was constructed on the basis of ${ }^{1} \mathrm{H}^{1} \mathrm{H}$ COSY and HMBC experiments. Namely, the ${ }^{1} \mathrm{H}^{1} \mathrm{H}$ COSY experiment suggested the existence of three partial structures, as shown as bold lines in Figure 6. Meanwhile, in its $\mathrm{HMBC}$ spectrum, long-rang correlations from $\delta_{\mathrm{H}} 4.27\left(\mathrm{H}-1^{\prime}\right)$ to $\delta_{\mathrm{C}} 70.4(\mathrm{C}-1) ; \delta_{\mathrm{H}} 4.31$ $\left(\mathrm{H}-1^{\prime \prime}\right)$ to $\delta_{\mathrm{C}} 69.6\left(\mathrm{C}-6^{\prime}\right)$ were observed. Finally, the NOE correlation between $\delta_{\mathrm{H}} 2.39,2.46\left(\mathrm{H}_{2}-2\right)$, and $\delta_{\mathrm{H}} 4.61(\mathrm{H}-5) ; 5.45(\mathrm{H}-3)$ and $\delta_{\mathrm{H}} 5.47(\mathrm{H}-5)$ found in the NOESY spectrum indicated the configuration in the 3-position was Z. Consequently, the structure of 5 was elucidated to be (3Z)-3-hexene-1,5-diol 1 -O- $\alpha$-L-arabinopyranosyl $(1 \rightarrow 6)-\beta$-D-glucopyranoside.

Table 5. ${ }^{1} \mathrm{H}$ and ${ }^{13} \mathrm{C}-\mathrm{NMR}$ data for 5 in $\mathrm{CD}_{3} \mathrm{OD}$.

\begin{tabular}{cccccc}
\hline No. & $\delta_{\mathbf{C}}$ & $\delta_{\mathbf{H}}(\boldsymbol{J}$ in Hz) & No. & $\delta_{\mathbf{C}}$ & $\mathcal{\delta}_{\mathbf{H}}(\boldsymbol{J}$ in $\mathbf{H z})$ \\
\hline 1 & 70.4 & $3.61(\mathrm{dd}, 6.5,11.5)$ & $4^{\prime}$ & 71.7 & $3.33(\mathrm{dd}, 8.0,8.0)$ \\
& & $3.86(\mathrm{~m}$, overlapped) & $5^{\prime}$ & 76.9 & $3.43(\mathrm{~m})$ \\
2 & 29.3 & $2.39(\mathrm{~m})$ & $6^{\prime}$ & 69.6 & $3.72(\mathrm{dd}, 5.5,11.0)$ \\
3 & 127.4 & $2.46(\mathrm{~m})$ & & & $4.09(\mathrm{dd}, 2.0,11.0)$ \\
4 & 137.0 & $5.45(\mathrm{~m})$ & $1^{\prime \prime}$ & 105.2 & $4.31(\mathrm{~d}, 6.5)$ \\
5 & 64.4 & $5.47(\mathrm{~m})$ & $2^{\prime \prime}$ & 72.4 & $3.58(\mathrm{dd}, 6.5,9.0)$ \\
6 & 23.9 & $1.20(\mathrm{~d}, 6.0)$ & $3^{\prime \prime}$ & 74.2 & $3.52(\mathrm{dd}, 3.5,9.0)$ \\
$1^{\prime}$ & 104.4 & $4.27(\mathrm{~d}, 7.5)$ & $4^{\prime \prime}$ & 69.5 & $3.80(\mathrm{~m})$ \\
$2^{\prime}$ & 75.1 & $3.17(\mathrm{dd}, 7.5,8.0)$ & & & $3.53(\mathrm{dd}, 2.0,12.5)$ \\
$3^{\prime}$ & 78.0 & $3.34(\mathrm{dd}, 8.0,8.0)$ & & & \\
\hline
\end{tabular}




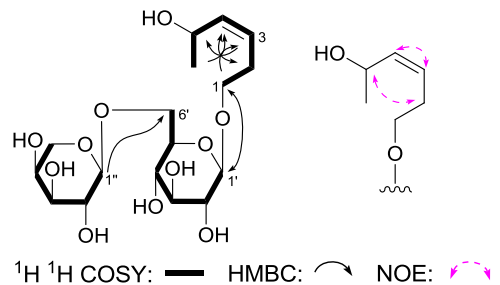

Figure 6. The main ${ }^{1} \mathrm{H}^{1} \mathrm{H}$ COSY, HMBC, and NOE correlations of 5.

Furthermore, inhibitory effects of all fractions obtained from $70 \%$ EtOH extract of G. acuta and the abovementioned isolates on motility of mouse isolated intestine tissue were determined by using the same method as reported previously $[4,28]$. As results, all of the test samples showed no significant changing on isolated intestinal tissue contraction frequency, while 70\% EtOH extract of G. acut, 95\% EtOH eluate from D101 macroporous resin $\mathrm{CC}, \mathrm{CHCl}_{3}$ layer, as well as compounds 5, 7, 10, 12-14, 16, 17, 31, and 32 displayed significant inhibitory effects on contraction tension (Table 6).

From the whole plants of G. acuta, two types of monoterpenes, iridoid- (1, 22-26) and secoiridoid-type (7-21) monoterpenes were obtained. Structure-activity relationship analysis revealed that iridoid-type monoterpenes showed no significant effect on contraction tension. However, secoiridoid-type monoterpenes, such as $\mathbf{7 , 1 0 , 1 2 - 1 4 , 1 6 , 1 7 ~ h a d ~ s t r o n g ~ i n h i b i t o r y ~ e f f e c t . ~ F u r t h e r m o r e , ~}$ when an olefin functional existed between the 5- and 6-positions $(\mathbf{2}, \mathbf{2 0}, \mathbf{2 1})$, or $\mathrm{H}-5$ was substituted by hydroxyl $(\mathbf{1 8}, \mathbf{1 9})$, the bioactivity disappeared.

Meanwhile, comparing the inhibitory effect on contraction tension of 8-O-4'- (27), 7-O-4'- (28-30) with those of 7-O-9'-type $(\mathbf{3 1}, \mathbf{3 2})$ lignan, we found that 7-O-9'-type $(\mathbf{3 1}, \mathbf{3 2})$ lignan displayed strong inhibitory bioactivity on contraction tension.

Table 6. Inhibitory effects of fractions and compounds 1-32 on motility of mouse isolated intestine tissue.

\begin{tabular}{ccccccc}
\hline & \multicolumn{2}{c}{ Intestine Motility (\%) } & & \multicolumn{2}{c}{ Intestine Motility (\%) } \\
\cline { 2 - 3 } & Relative Tension & Relative Frequency & & Relative Tension & Relative Frequency \\
\hline $\mathrm{N}$ & $100.0 \pm 4.0$ & $100.0 \pm 7.3$ & $\mathbf{1 4}$ & $90.1 \pm 2.6^{*}$ & $102.1 \pm 1.9$ \\
$\mathrm{P}$ & $74.1 \pm 9.3^{*}$ & $82.7 \pm 5.3^{*}$ & $\mathbf{1 5}$ & $88.9 \pm 5.0$ & $104.7 \pm 2.3$ \\
$\mathrm{~A}$ & $83.1 \pm 3.4^{*}$ & $97.5 \pm 3.6$ & $\mathbf{1 6}$ & $80.5 \pm 6.1^{*}$ & $102.1 \pm 5.8$ \\
$\mathrm{~B}$ & $93.9 \pm 4.2$ & $103.2 \pm 3.1$ & $\mathbf{1 7}$ & $64.9 \pm 7.1^{* *}$ & $94.3 \pm 2.0$ \\
$\mathrm{C}$ & $74.5 \pm 3.8^{* * *}$ & $99.3 \pm 5.3$ & $\mathbf{1 8}$ & $88.2 \pm 4.9$ & $86.4 \pm 8.8$ \\
$\mathrm{D}$ & $78.4 \pm 3.5^{* * *}$ & $103.2 \pm 4.4$ & $\mathbf{1 9}$ & $93.7 \pm 7.1$ & $100.3 \pm 2.4$ \\
$\mathbf{1}$ & $93.2 \pm 3.5$ & $102.0 \pm 3.7$ & $\mathbf{2 0}$ & $85.8 \pm 5.0$ & $98.2 \pm 1.4$ \\
$\mathbf{2}$ & $88.2 \pm 3.4$ & $97.7 \pm 4.9$ & $\mathbf{2 1}$ & $86.8 \pm 4.6$ & $102.2 \pm 3.2$ \\
$\mathbf{3}$ & $88.8 \pm 5.1$ & $113.0 \pm 14.0$ & $\mathbf{2 2}$ & $89.6 \pm 4.4$ & $112.0 \pm 8.1$ \\
$\mathbf{4}$ & $100.4 \pm 2.0$ & $100.4 \pm 2.0$ & $\mathbf{2 3}$ & $91.2 \pm 2.7$ & $103.6 \pm 7.4$ \\
$\mathbf{5}$ & $81.6 \pm 2.9^{* *}$ & $98.5 \pm 0.9$ & $\mathbf{2 4}$ & $91.0 \pm 3.5$ & $101.0 \pm 4.4$ \\
$\mathbf{6}$ & $89.5 \pm 7.0$ & $100.9 \pm 5.5$ & $\mathbf{2 5}$ & $91.0 \pm 3.8$ & $102.1 \pm 2.6$ \\
$\mathbf{7}$ & $82.9 \pm 7.0^{*}$ & $100.8 \pm 2.6$ & $\mathbf{2 6}$ & $84.8 \pm 4.7$ & $105.9 \pm 1.6$ \\
$\mathbf{8}$ & $86.6 \pm 4.6$ & $85.2 \pm 3.0$ & $\mathbf{2 7}$ & $77.4 \pm 7.4$ & $100.8 \pm 5.6$ \\
$\mathbf{9}$ & $91.8 \pm 3.3$ & $100.7 \pm 2.3$ & $\mathbf{2 8}$ & $76.7 \pm 10.1$ & $97.4 \pm 3.5$ \\
$\mathbf{1 0}$ & $75.5 \pm 6.4^{* *}$ & $99.7 \pm 1.4$ & $\mathbf{2 9}$ & $90.4 \pm 9.0$ & $102.6 \pm 1.0$ \\
$\mathbf{1 1}$ & $88.9 \pm 5.0$ & $98.4 \pm 3.3$ & $\mathbf{3 0}$ & $91.0 \pm 6.3$ & $95.9 \pm 3.3$ \\
$\mathbf{1 2}$ & $75.7 \pm 9.1^{*}$ & $96.6 \pm 4.6$ & $\mathbf{3 1}$ & $79.1 \pm 3.7^{* *}$ & $102.3 \pm 5.2$ \\
$\mathbf{1 3}$ & $84.9 \pm 4.0^{*}$ & $100.3 \pm 1.4$ & $\mathbf{3 2}$ & $82.4 \pm 4.7^{*}$ & $92.2 \pm 1.3$ \\
\hline
\end{tabular}

Values are the means \pm standard error of measurement, significantly different from the control group, ${ }^{*} p<0.05$, ${ }^{* *} p<0.01,{ }^{* * *} p<0.001, n=6$. Normal (N): isolated intestine tissue; Positive control (P): Loperamide hydrochloride, final concentration was $10 \mu \mathrm{M}$. A: G. acuta 70\% EtOH extract; B: $\mathrm{H}_{2} \mathrm{O}$ eluate from D101 resin for extract; C: $95 \%$ EtOH eluate from D101 resin for extract; D: $\mathrm{CHCl}_{3}$ layer for extract, and their final concentration was $100 \mu \mathrm{g} / \mathrm{mL}$. Compounds 1-32: final concentration was $40 \mu \mathrm{M}$. Tension and frequency of normal group was set as $100 \%$, relative tension, and frequency were calculated as: (sample/normal) $\times 100 \%$. 


\section{Experimental}

\subsection{General}

Physical data was obtained by using the following instruments: UV and IR spectra were determined on a Varian Cary 50 UV-VIS (Varian, Inc., Hubbardsdon, MA, USA) and Varian 640-IR FT-IR spectrophotometer (Varian Australia Pty Ltd., Mulgrave, Australia), respectively. Optical rotations were obtained on a Rudolph Autopol ${ }^{\circledR}$ IV automatic polarimeter $(1=50 \mathrm{~mm})$ (Rudolph Research Analytical, 55 Newburgh Road, Hackettstown, NJ, 07840 USA). NMR spectra were run on a Bruker 500 MHz NMR spectrometer (Bruker BioSpin AG Industriestrasse 26 CH-8117, Fällanden, Switzerland) at $500 \mathrm{MHz}$ for ${ }^{1} \mathrm{H}$ and $125 \mathrm{MHz}$ for ${ }^{13} \mathrm{CNMR}$ (internal standard: TMS). Negative-ion HRESI-TOF-MS were recorded on an Agilent 6520 Accurate-Mass Q-Tof LC/MS spectrometer (Agilent Corp., Santa Clara, CA, USA). Column chromatographies (CC) were performed on macroporous resin D101 (Haiguang Chemical Co., Ltd., Tianjin, China), silica gel (74-149 $\mu \mathrm{m}$, Qingdao Haiyang Chemical Co., Ltd., Qingdao, China), and Sephadex LH-20 (Ge Healthcare Bio-Sciences, Uppsala, Sweden). Preparative high-performance liquid chromatography (PHPLC) column, cosmosil 5C 18 -MS-II (20 mm i.d. $\times 250 \mathrm{~mm}$, Nakalai Tesque, Inc., Tokyo, Japan) were used to isolate the compounds.

\subsection{Plant Material}

The whole plants of Gentianella acuta (Michx.) Hulten were collected from Alxa Youqi, Inner Mongolia Autonomous region, China in September 2013, and identified by Dr. Li Tianxiang (Experiment Teaching Department, Tianjin University of Traditional Chinese Medicine). The voucher specimen was deposited at the Academy of Traditional Chinese Medicine of Tianjin University of TCM.

\subsection{Extraction and Isolation}

The whole plants of G. acuta $(3.0 \mathrm{~kg})$ were cut and refluxed with $70 \%$ ethanol-water. Then the $70 \%$ EtOH extract (868.5 g) was partitioned in a $\mathrm{CHCl}_{3}-\mathrm{H}_{2} \mathrm{O}$ mixture $(1: 1, v / v)$. The $\mathrm{H}_{2} \mathrm{O}$ layer (670.0 g) was subjected to D101 macroporous resin $\mathrm{CC}\left(\mathrm{H}_{2} \mathrm{O} \rightarrow 95 \% \mathrm{EtOH} \rightarrow\right.$ acetone). As a result, $\mathrm{H}_{2} \mathrm{O}$ (332.4 g), 95\% EtOH (294.9 g), and acetone (5.1 g) eluates were obtained.

The $95 \%$ EtOH eluate $(200.0 \mathrm{~g})$ was subjected to silica gel CC $\left[\mathrm{CHCl}_{3} \rightarrow \mathrm{CHCl}_{3}-\mathrm{MeOH}(100: 1 \rightarrow\right.$ $100: 5, v / v) \rightarrow \mathrm{CHCl}_{3}-\mathrm{MeOH}-\mathrm{H}_{2} \mathrm{O}(10: 3: 1 \rightarrow 7: 3: 1 \rightarrow 6: 4: 1, v / v / v$, lower layer $\left.)\right]$ to give 16 fractions (Fr. 1-Fr. 16). Fraction 7 (25.7 g) was centrifugated (MeOH), and two fractions (Fr. 7-1-Fr. 7-2) were yielded. Fraction 7-2 (9.8 g) was separated by PHPLC $\left[\mathrm{CH}_{3} \mathrm{CN}-\mathrm{H}_{2} \mathrm{O}(18: 82 \rightarrow 35: 65 \rightarrow 42: 58\right.$, $v / v)+1 \% \mathrm{HAc}]$ to give 27 fractions (Fr. 7-2-1-Fr. 7-2-27). Fraction 7-2-2 (70.6 mg) was purified by PHPLC [MeOH-H $\left.\mathrm{H}_{2} \mathrm{O}(22: 78, v / v)\right]$, and 2,3-dihydroxy-1-(4-hydroxy-3-methoxyphenyl)-propan-1-one $(6,5.9 \mathrm{mg})$ was given. Fraction 7-2-8 $(46.3 \mathrm{mg})$ was isolated by PHPLC $\left[\mathrm{CH}_{3} \mathrm{CN}-\mathrm{H}_{2} \mathrm{O}(20: 80, v / v)+\right.$ $1 \% \mathrm{HAc}]$ to gain $(7 R, 8 S)$-erythro-4,7,9,9'-tetrahydroxy-3,3'-dimethoxy-8-O-4' -neolignan $(27,6.0 \mathrm{mg})$. Fraction $9(15.0 \mathrm{~g})$ was subjected to Sephadex LH-20 CC $\left[\mathrm{CHCl}_{3}-\mathrm{MeOH}(1: 1, v / v)\right]$ to yield seven fractions (Fr. 9-1-Fr. 9-7). Fraction 9-4 (7.3 g) was separated by PHPLC $\left[\mathrm{CH}_{3} \mathrm{CN}-\mathrm{H}_{2} \mathrm{O}(22: 78\right.$ $\rightarrow 30: 70 \rightarrow 45: 55, v / v)+1 \%$ HAc], as a result, 12 fractions (Fr. 9-4-1-Fr. 9-4-12) were obtained. Fraction 9-4-2 (962.8 mg) was purified by PHPLC [MeOH- $\mathrm{H}_{2} \mathrm{O}(23: 77, v / v)+1 \% \mathrm{HAc}$ ] to give gentiiridoside B (2,14.8 mg). Fraction 9-4-9 (277.1 mg) was centrifuged (MeOH), and two fractions (Fr. 9-4-9-1-Fr. 9-4-9-2) were gained. Fraction 9-4-9-2 (199.7 mg) was isolated by PHPLC [MeOH-H $2 \mathrm{O}$ $(45: 55, v / v)+1 \% \mathrm{HAc}]$ to yield plucheoside $\mathrm{D}_{3}(29,8.8 \mathrm{mg})$. Fraction $11(20.0 \mathrm{~g})$ was subjected to PHPLC $\left[\mathrm{CH}_{3} \mathrm{CN}-\mathrm{H}_{2} \mathrm{O}(15: 85 \rightarrow 25: 75 \rightarrow 42: 58, v / v)+1 \%\right.$ HAc], and 29 fractions (Fr. 11-1-Fr. $11-29)$ were given. Fraction 11-5 (631.3 mg) was separated by PHPLC $\left[\mathrm{CH}_{3} \mathrm{CN}-\mathrm{H}_{2} \mathrm{O}(10: 90, v / v)+\right.$ 1\% HAc], and 8-epiloganin (24, $29.7 \mathrm{mg})$ was yielded. Fraction 11-6 (388.0 mg) was isolated by PHPLC $\left[\mathrm{CH}_{3} \mathrm{CN}-\mathrm{H}_{2} \mathrm{O}(11: 89, v / v)+1 \% \mathrm{HAc}\right]$ to yield gentilignanoside $\mathrm{A}(3,9.2 \mathrm{mg}), \mathrm{loganin}$ (23, $143.5 \mathrm{mg})$, and berchemol-4'-O- $\beta$-D-glucoside $(32,84.5 \mathrm{mg})$. Fraction 11-12 (660.5 mg) was centrifugated $(\mathrm{MeOH})$ and further purified by PHPLC $\left[\mathrm{MeOH}-\mathrm{H}_{2} \mathrm{O}(35: 65, v / v)+1 \%\right.$ HAc] to give (1R)-2,2,3-trimethyl-4-hydroxymethylcyclopent-3-ene-1-methyl-O- $\beta$-D-glucopyranoside (4, $11.5 \mathrm{mg})$. 
Fraction 11-17 (790.7 mg) was isolated by PHPLC [MeOH- $\mathrm{H}_{2} \mathrm{O}(42: 58, v / v)+1 \%$ HAc] to obtain six fractions (Fr. 11-17-1-Fr. 11-17-6). Fraction 11-17-1 (97.1 mg) was purified by PHPLC $\left[\mathrm{CH}_{3} \mathrm{CN}-\mathrm{H}_{2} \mathrm{O}\right.$ $(24: 76, v / v)+1 \% \mathrm{HAc}$ to gain swertiaside (26, $79.0 \mathrm{mg})$. Fraction 11-21 (376.1 mg) was separated by PHPLC [MeOH- $\mathrm{H}_{2} \mathrm{O}(42: 58, v / v)+1 \%$ HAc] to afford (7S,8R)-9'-methoxy-dehydrodiconiferyl alcohol 4-O- $\beta$-D-glucopyranoside (30, $17.4 \mathrm{mg})$. Fraction 13 (20.0 g) was subjected to PHPLC [MeOH- $\mathrm{H}_{2} \mathrm{O}$ $(35: 65 \rightarrow 45: 55 \rightarrow 55: 45, v / v)+1 \%$ HAc], and 20 fractions (Fr. 13-1-Fr. 13-20) were yielded. Fraction 13-11 (1.9 g) was centrifuged (MeOH) to obtain two fractions (Fr. 13-11-1-Fr. 13-11-2). Fraction 13-11-1 $(1.0 \mathrm{~g})$ was isolated by PHPLC $\left[\mathrm{CH}_{3} \mathrm{CN}-\mathrm{H}_{2} \mathrm{O}(16: 84, v / v)+1 \% \mathrm{HAc}\right]$, as a result, eleven fractions (Fr. 13-11-1-1-Fr. 13-11-1-11) were given. Fraction 13-11-1-9 (117.1 mg) were purified by Sephadex LH-20 $\mathrm{CC}(\mathrm{MeOH})$ and PHPLC [MeOH- $\left.\mathrm{H}_{2} \mathrm{O}(35: 65, v / v)+1 \% \mathrm{HAc}\right]$ to afford $5 \alpha$-carboxystrictosidine (12, $26.2 \mathrm{mg})$. Fraction 13-12 (501.4 mg) was isolated by PHPLC $\left[\mathrm{CH}_{3} \mathrm{CN}-\mathrm{H}_{2} \mathrm{O}(18: 82, v / v)+1 \% \mathrm{HAc}\right]$ and to yield $(E)$-aldosecologanin $(\mathbf{1 1}, 38.2 \mathrm{mg})$. Fraction 13-17 $(504.0 \mathrm{mg})$ was purified by PHPLC $\left[\mathrm{CH}_{3} \mathrm{CN}-\mathrm{H}_{2} \mathrm{O}(22: 78, v / v)+1 \% \mathrm{HAc}\right]$ to yield gentiiridoside A $(\mathbf{1}, 265.0 \mathrm{mg})$. Fraction 14 (15.3 g) was subjected to PHPLC $\left[\mathrm{CH}_{3} \mathrm{CN}-\mathrm{H}_{2} \mathrm{O}(15: 85 \rightarrow 25: 75, v / v)+1 \% \mathrm{HAc}\right]$, and 20 fractions (Fr. 14-1-Fr. 14-20) were obtained. Fraction 14-1 (1.4 g) was separated by PHPLC $\left[\mathrm{CH}_{3} \mathrm{CN}-\mathrm{H}_{2} \mathrm{O}(9: 91, v / v)+1 \%\right.$ HAc] to gain 14 fractions (Fr. 14-1-1-Fr. 14-1-14). Fraction 14-1-2 (32.1 mg) was purified by Sephadex LH-20 CC (MeOH) and finally by PHPLC $\left[\mathrm{CH}_{3} \mathrm{CN}-\mathrm{H}_{2} \mathrm{O}(7: 93, v / v)\right]$ to give (3Z)-3-hexene-1,5-diol 1-O- $\alpha$-L-arabinopyranosyl $(1 \rightarrow 6)-\beta$-D-glucopyranoside $(5,2.8 \mathrm{mg})$.

The $\mathrm{CHCl}_{3}$ layer $\left(50.0 \mathrm{~g}\right.$, Fr. C) was subjected to Silica gel CC $\left[\mathrm{CHCl}_{3}-\mathrm{MeOH}(100: 2 \rightarrow 100: 3\right.$ $\rightarrow 100: 5, v / v) \rightarrow \mathrm{CHCl}_{3}-\mathrm{MeOH}-\mathrm{H}_{2} \mathrm{O}(10: 3: 1, v / v / v$, lower layer) $\rightarrow \mathrm{MeOH}]$, and eight fractions (Fr. C-1-Fr. C-8) were yielded. Fraction C-5 (1.1 g) was separated by Sephadex $\mathrm{LH}-20 \mathrm{CC}$ [MeOH-CH $\mathrm{Cl}_{2}$ $(1: 1, v / v)]$ to gain three fractions (Fr. C-5-1-Fr. C-5-3). Fraction C-5-2 $(110.0 \mathrm{mg})$ was purified by PHPLC [MeOH- $\left.\mathrm{H}_{2} \mathrm{O}(45: 55, v / v)\right]$ to afford $(7 S, 8 R)$-dehydrodiconiferyl alcohol $(\mathbf{2 8}, 13.3 \mathrm{mg})$ and (-)-berchemol $(31,14.6 \mathrm{mg})$. Fraction C-7 $(9.0 \mathrm{~g})$ was isolated by ODS CC $\left[\mathrm{MeOH}-\mathrm{H}_{2} \mathrm{O}(20: 80 \rightarrow 30: 70 \rightarrow 40: 60 \rightarrow\right.$ $50: 50, v / v) \rightarrow \mathrm{MeOH}$, and eight fractions (Fr. C-7-1-Fr. C-7-8) were obtained. Fraction C-7-2 (351.4 mg) was purified by PHPLC [ $\left.\mathrm{MeOH}-\mathrm{H}_{2} \mathrm{O}(32: 68, v / v)+1 \% \mathrm{HAc}\right]$ to give gentiopicroside $(20,15.9$ mg). Fraction C-7-6 (486.4 g) was subjected to Sephadex LH-20 CC [MeOH-C $\left.\mathrm{CH}_{2} \mathrm{Cl}_{2}(1: 1, v / v)\right]$ and PHPLC [MeOH- $\left.\mathrm{H}_{2} \mathrm{O}(50: 50, v / v)+1 \% \mathrm{HAc}\right]$ to afford trifloroside $(\mathbf{1 7}, 16.0 \mathrm{mg})$.

Compounds 7-10, 13-16, 18, 19, 21, 22 and 25 were obtained and identified by using the method reported previously $[2,3]$.

Gentiiridoside $A$ (1): White powder; $[\alpha]_{\mathrm{D}}^{25}-90.0^{\circ}\left(c\right.$ 0.14, MeOH); UV $\lambda_{\max }(\mathrm{MeOH}) \mathrm{nm}(\log \varepsilon): 229$ (4.46), 285 (3.53); IR $v_{\max }$ (KBr): 3372, 2929, 1712, 1635, 1588, 1486, 1372, 1264, 1201, 1154, 1078, 1017, 902, $873 \mathrm{~cm}^{-1} ;{ }^{1} \mathrm{H}-\mathrm{NMR}\left(\mathrm{CD}_{3} \mathrm{OD}, 500 \mathrm{MHz}\right)$ and ${ }^{13} \mathrm{C}-\mathrm{NMR}\left(\mathrm{CD}_{3} \mathrm{OD}, 125 \mathrm{MHz}\right)$ data, see Table 1. HRESI-TOF-MS negative-ion mode $m / z 777.2261[\mathrm{M}-\mathrm{H}]^{-}\left(\right.$calcd for $\mathrm{C}_{36} \mathrm{H}_{41} \mathrm{O}_{19}, 777.2248$ ).

Gentiiridoside B (2): White powder; $[\alpha]_{\mathrm{D}}^{25}-36.7^{\circ}$ (c 0.12, MeOH); UV $\lambda_{\max }(\mathrm{MeOH}) \mathrm{nm}(\log \varepsilon): 231$ (4.26, sh), 273 (4.08, sh); IR $v_{\max }(\mathrm{KBr}) 3357,2924,1705,1609,1518,1457,1418,1375,1272,1209,1074$, $1024 \mathrm{~cm}^{-1} ;{ }^{1} \mathrm{H}-\mathrm{NMR}$ (DMSO- $d_{6}, 500 \mathrm{MHz}$ ) and ${ }^{13} \mathrm{C}-\mathrm{NMR}$ (DMSO- $d_{6}, 125 \mathrm{MHz}$ ) data see Table 2. HRESI-TOF-MS negative-ion mode $m / z 679.1023$ [M - H] $]^{-}$(calcd for $\mathrm{C}_{28} \mathrm{H}_{39} \mathrm{O}_{18}, 679.1033$ ).

Gentilignanoside $A$ (3): White powder; $[\alpha]_{\mathrm{D}}^{25}-36.0^{\circ}$ (c 0.10, MeOH); $\mathrm{CD}(c 0.0018 \mathrm{M}, \mathrm{MeOH})$ mdeg $\left(\lambda_{\mathrm{nm}}\right):-3.8(278),-16.9(232),-27.6(206) ; \mathrm{UV} \lambda_{\max }(\mathrm{MeOH}) \mathrm{nm}(\log \varepsilon): 226$ (4.32), 275 (3.73); IR $v_{\max }(\mathrm{KBr}): 3368,2937,1613,1514,1463,1425,1324,1266,1224,1158,1115,1073,1026 \mathrm{~cm}^{-1} ;{ }^{1} \mathrm{H}-\mathrm{NMR}$ $\left(\mathrm{CD}_{3} \mathrm{OD}, 500 \mathrm{MHz}\right)$ and ${ }^{13} \mathrm{C}-\mathrm{NMR}\left(\mathrm{CD}_{3} \mathrm{OD}, 125 \mathrm{MHz}\right)$ data see Table 3. HRESI-TOF-MS negative-ion mode $m / z 679.1023[\mathrm{M}-\mathrm{H}]^{-} \mathrm{m} / z 567.2083[\mathrm{M}-\mathrm{H}]^{-}$(calcd for $\mathrm{C}_{27} \mathrm{H}_{33} \mathrm{O}_{13}, 567.2083$ ).

(1R)-2,2,3-Trimethyl-4-hydroxymethylcyclopent-3-ene-1-methyl-O- $\beta$-D-glucopyranoside (4): White powder; $[\alpha]_{\mathrm{D}}^{25}-43.9^{\circ}$ (c 0.12, MeOH); IR $v_{\max }(\mathrm{KBr}): 3367,2927,1636,1576,1436,1286,1161,1076,1038 \mathrm{~cm}^{-1}$. ${ }^{1} \mathrm{H}-\mathrm{NMR}\left(\mathrm{CD}_{3} \mathrm{OD}, 500 \mathrm{MHz}\right)$ and ${ }^{13} \mathrm{C}-\mathrm{NMR}\left(\mathrm{CD}_{3} \mathrm{OD}, 125 \mathrm{MHz}\right)$ data see Table 4. HRESI-TOF-MS negative-ion mode $m / z 377.1814[\mathrm{M}+\mathrm{COOH}]^{-}$(calcd for $\mathrm{C}_{17} \mathrm{H}_{29} \mathrm{O}_{9}$, 377.1817). 
(3Z)-3-Hexene-1,5-diol 1-O- $\alpha$-L-arabinopyranosyl $(1 \rightarrow 6)-\beta$-D-glucopyranoside (5): White powder; $[\alpha]_{\mathrm{D}}^{25}$ $-14.5^{\circ}$ (c 0.11, MeOH); IR $v_{\max }(\mathrm{KBr}): 3364,2966,2920,1593,1419,1370,1258,1166,1047,1009 \mathrm{~cm}^{-1}$; ${ }^{1} \mathrm{H}-\mathrm{NMR}\left(\mathrm{CD}_{3} \mathrm{OD}, 500 \mathrm{MHz}\right)$ and ${ }^{13} \mathrm{C}-\mathrm{NMR}\left(\mathrm{CD}_{3} \mathrm{OD}, 125 \mathrm{MHz}\right)$ data see Table 5. HRESI-TOF-MS negative-ion mode $m / z 455.1773[\mathrm{M}+\mathrm{COOH}]^{-}$(calcd for $\mathrm{C}_{18} \mathrm{H}_{31} \mathrm{O}_{13}$, 455.1770).

Enzymatic Hydrolysis of 4 A solution of $4(6.0 \mathrm{mg})$ in $\mathrm{H}_{2} \mathrm{O}(2.0 \mathrm{~mL})$ was reacted with $\beta$-glucosidase (6.0 mg, Almond, Sigma-Aldrich, Co. 3050 Spruce Street, St. Louis, MO, 63103 USA) at $37{ }^{\circ} \mathrm{C}$ for $2.5 \mathrm{~h}$. Then the reaction mixture was extracted with EtOAc. And the residue from EtOAc solvent was subjected to Silica gel $\mathrm{CC}\left[\mathrm{CHCl}_{3}-\mathrm{MeOH}(100: 5, v / v)\right]$, as a result, the aglycon, (1R)-2,2,3-trimethyl-4-hydroxymethylcyclopent-3-ene-1-methanol (4a, $2.8 \mathrm{mg}, 93.33 \%$ ) was yielded.

(1R)-2,2,3-Trimethyl-4-hydroxymethylcyclopent-3-ene-1-methanol (4a): White powder; $[\alpha]_{\mathrm{D}}^{25}-6.5^{\circ}(c 0.09$, $\left.\mathrm{CHCl}_{3}\right)$; IR $v_{\max }(\mathrm{KBr}): 3318,2953,2925,2867,1717,1576,1462,1437,1380,1240,1179,1118$, 1087, $999 \mathrm{~cm}^{-1} ;{ }^{1} \mathrm{H}-\mathrm{NMR}\left(\mathrm{CD}_{3} \mathrm{OD}, 500 \mathrm{MHz}\right)$ and ${ }^{13} \mathrm{C}-\mathrm{NMR}\left(\mathrm{CD}_{3} \mathrm{OD}, 125 \mathrm{MHz}\right)$ data see Table 4. HRESI-TOF-MS negative-ion mode $m / z 170.1423[\mathrm{M}-\mathrm{H}]^{-}$(calcd for $\mathrm{C}_{10} \mathrm{H}_{17} \mathrm{O}_{2}, 170.1433$ ).

Acid Hydrolysis of 1-5 The solution of compounds 1-5 (each $2.0 \mathrm{mg})$ in $1 \mathrm{M} \mathrm{HCl}(1.0 \mathrm{~mL})$ was treated by using the same method as described in reference [4]: They were heated under reflux for $3 \mathrm{~h}$. The reaction mixture was then analyzed by $\mathrm{CH}_{3} \mathrm{CN}-\mathrm{H}_{2} \mathrm{O}(75: 25, v / v$; flow rate $0.7 \mathrm{~mL} / \mathrm{min})$. As results, D-glucose was detected from the aqueous phase of 1-5, and L-arabinose was found from that of 5 by comparison of its retention time and optical rotation with those of the authentic sample, D-glucose $\left(t_{\mathrm{R}} 16.8 \mathrm{~min}\right.$ (positive)) and L-arabinose $\left(t_{\mathrm{R}} 13.1 \mathrm{~min}\right.$ (positive)), respectively.

\subsection{Inhibitory Effects of Fractions and Compounds 1-32 on the Motility of Mouse Isolated Intestine Tissue}

Inhibitory effects of fractions and compounds 1-32 on motility of mice isolated intestine tissue were determined by using the similar method as we reported previously [4,28]: Mice were fasted for $12 \mathrm{~h}$ before experiments, intestinal tissue were collected immediately. The Maxwell bath was filled with $10 \mathrm{~mL}$ of Tyrode's solution (one liter contains: $\mathrm{NaCl} 8.0 \mathrm{~g}, \mathrm{CaCl}_{2} 0.2$ g, $\mathrm{KCl} 0.2 \mathrm{~g}, \mathrm{MgCl}_{2} 0.1 \mathrm{~g}, \mathrm{NaHCO}_{3}$ $1.0 \mathrm{~g}, \mathrm{KH}_{2} \mathrm{PO}_{4} 0.05 \mathrm{~g}$, glucose $\left.1.0 \mathrm{~g}, \mathrm{pH} 7.4\right)$ and maintained at a constant temperature $\left(37.0 \pm 0.5^{\circ} \mathrm{C}\right)$, and bubbled with $95 \% \mathrm{O}_{2}$ and $5 \% \mathrm{CO}_{2}$ gas. The intestinal tissue was fixed on bottom hook in and the other end was connected to an isometric tension transducer. Samples in DMSO solution were added after $15 \mathrm{~min}$ to equilibrate incubation, the final DMSO concentration was $0.1 \%$, and the final concentration of fractions and compounds was $100 \mu \mathrm{g} / \mathrm{mL}$ and $40 \mu \mathrm{M}$, respectively. The mean tension and frequency of intestine muscle contractions were recorded for $1 \mathrm{~min}$ before and $4 \mathrm{~min}$ after drug additions using isolated tissue bath systems (Radnoti Glass Technology Inc., Monrovia, CA, 159901A, USA). Loperamide hydrochloride (Xi'an Janssen Pharmaceutical Ltd., Xi'an, China) was used as a positive control, and the final concentration was $10 \mu \mathrm{M}$.

Values are expressed as mean \pm S.D. All the grouped data were statistically performed with SPSS 11.0. Significant differences between means were evaluated by one-way analysis of variance (ANOVA) and Tukey's Studentized range test was used for post hoc evaluations. $p<0.05$ was considered to indicate statistical significance.

\section{Conclusions}

In summary, nineteen constituents, including five new ones, gentiiridosides A (1), B (2), gentilignanoside A (3), (1R)-2,2,3-trimethyl-4-hydroxymethylcyclopent-3-ene-1-methyl-O- $\beta$-Dglucopyranoside (4), and (3Z)-3-hexene-1,5-diol 1-O- $\alpha$-L-arabinopyranosyl( $1 \rightarrow 6)$ - $\beta$-D-glucopyranoside (5) were obtained from the whole plants of G. acuta in our on-going program of screening the phytochemical and bioactive constituents. Among the known isolates, 6, 11, 12, 17, 23, and 27-32 were isolated from the genus firstly. The structures of them were elucidated by chemical and spectroscopic methods.

Furthermore, the inhibitory effects on motility of mouse isolated intestine tissue of the above mentioned compounds and other thirteen isolates (7-10, 13-16, 18, 19, 21, 22 and 25) previously 
obtained in the plant were analyzed. Resultingly, 5, 7, 10, 12, 14, 16, 17, 31, and 32 displayed significant inhibitory effects on contraction tension. On the other hand, structure-activity relationship analysis revealed that the inhibitory effect of secoiridoid-type monoterpenes was stronger than that of iridoid-type monoterpenes, and 7-O-9'-type lignan displayed stronger inhibitory bioactivity than $8-O-4^{\prime}$ - and 7-O-4'-type lignan.

Our previous [4-6] and present research results suggested that the chemical constituents in G. acuta were xanthones, monoterpenes, lignans, and phenolic acids. Among them, xanthones and part of the monoterpenes were major bioactivity substances of G. acuta. These results suggested that G. acuta and its constituents have potential value in discovering new medicines for abnormal intestinal motility.

Acknowledgments: This work was financially supported by programs for National Natural Science Foundation of China $(21405113,81673688)$, Important Drug Development Fund, Ministry of Science and Technology of China (2017ZX09305002-002)

Author Contributions: Yi Zhang and Tao Wang designed the research and wrote the manuscript; Zhijuan Ding and Yanxia Liu performed the experimental work; Jingya Ruan and Shengcai Yang retrieved the literature; and Haiyang Yu and Meiling Chen perfected the language. All authors discussed, edited, and approved the final version.

Conflicts of Interest: The authors declare no conflicts of interest.

\section{References}

1. Wang, Z.; Wu, Q.; Yu, Y.; Yang, C.; Jiang, H.; Wang, Q.; Yang, B.; Kuang, H. Determination and pharmacokinetic study of four xanthones in rat plasma after oral administration of Gentianella acuta extract by UHPLC-ESI-MS/MS. J. Ethnopharmacol. 2015, 174, 261-269. [CrossRef] [PubMed]

2. Lv, L.J.; Wang, Y.F.; Yan, T.; Han, H.R.; Li, M.H. Preparation of swertianolin from Gentianella acuta and its effects on arrhythmia induced by aconitine in mice. Chin. J. New Drugs Clin. Rem. 2015, 34, 630-635.

3. Wang, Z.; Wu, G.; Liu, H.; Xing, N.; Sun, Y.; Zhai, Y.; Yang, B.; Kong, A.T.; Kuang, H.; Wang, Q. Cardioprotective effect of the xanthones from Gentianella acuta against myocardial ischemia/reperfusion injury in isolated rat heart. Biomed. Pharmacother. 2017, 93, 626-635. [CrossRef] [PubMed]

4. Liu, Y.; Ni, Y.; Ruan, J.; Qu, L.; Yu, H.; Han, L.; Zhang, Y.; Wang, T. Bioactive gentixanthone and gentichromone from the whole plants of Gentianella acuta (Michx.) Hulten. Fitoterapia 2016, 113, 164-169. [CrossRef] [PubMed]

5. Ni, Y.; Liu, Y.; Ruan, J.; Han, L.; Wang, T.; Zhang, Y. Isolation and identification of iridoidal glycosides from the whole plant of Gentianella acuta (II). Zhongguo Yaowuhuaxue Zazhi 2017, 27, 133-137.

6. Liu, Y.; Shi, W.; Ni, Y.; Chao, L.; Wang, T.; Zhang, Y. Isolation and identification of iridoidal glycosides from the whole plant of Gentianella acuta. J. Shenyang Pharma. Univ. 2016, 33, 702-705.

7. Baderschneider, B.; Winterhalter, P. Isolation and characterization of novel benzoates, cinnamates, flavonoids, and lignans from riesling wine and screening for antioxidant activity. J. Agric. Food Chem. 2001, 49, 2788-2798. [CrossRef] [PubMed]

8. Machida, K.; Sasaki, H.; Iijima, T.; Kikuchi, M. Studies on the constituents of Lonicera species. XVII. New iridoid glycosides of the stems and leaves of Lonicera japonica Thunb. Chem. Pharm. Bull. 2002, 50, 1041-1044. [CrossRef] [PubMed]

9. Ferrari, F.; Messana, I.; Botta, B.; De Mello, J.F. Constituents of Guettarda platypoda. J. Nat. Prod. 1986, 49, 1150-1151. [CrossRef]

10. Kim, J.A.; Son, N.S.; Son, J.K.; Jahng, Y.; Chang, H.W.; Jang, T.S.; Na, M.K.; Lee, S.H. Two new secoiridoid glycosides from the rhizomes of Gentiana scabra Bunge. Arch. Pharm. Res. 2009, 32, 863-867. [CrossRef] [PubMed]

11. Rho, T.; Jung, M.; Lee, M.W.; Chin, Y.W.; Yoon, K.D. Efficient methods for isolating five phytochemicals from Gentiana macrophylla using high-performance countercurrent chromatography. J. Sep. Sci. 2016, 39, 4723-4731. [CrossRef] [PubMed]

12. Calis, I.; Lahloub, M.F.; Sticher, O. Loganin, loganic acid and periclymenoside, a new biosidic ester iridoid glucoside from Loniceva peviclymenum L. (Capvifoliaceae). Helv. Chim. Acta 1984, 67, 160-165. [CrossRef]

13. Balázs, B.; Tóth, G.; Duddeck, H.; Soliman, H.S. Iridoid and lignan glycosides from Citharexylum spinosum L. Nat. Prod. Res. 2006, 20, 201-205. [CrossRef] [PubMed] 
14. Ikeshiro, Y.; Tomita, Y. A new iridoid glucoside of Swertia japonica. Planta Med. 1984, 50, 485-488. [CrossRef] [PubMed]

15. Huo, C.H.; Liang, H.; Zhao, Y.Y.; Wang, B.; Zhang, Q.Y. Neolignan glycosides from Symplocos caudata. Phytochemistry 2008, 69, 788-795. [CrossRef] [PubMed]

16. Della, G.M.; Molinaro, A.; Monaco, P.; Previtera, L. Neolignans from Arum ztalzcum. Phytochemistry 1994, 35, 777-779.

17. Iida, N.; Inatomi, Y.; Murata, H.; Murata, J.; Lang, F.A.; Tanaka, T.; Nakanishi, T.; Inada, A. New phenylpropanoid glycosides from Juniperus communis var. Depressa. Chem. Pharm. Bull. 2010, 58, 742-746. [CrossRef] [PubMed]

18. Xiao, H.H.; Dai, Y.; Wong, M.S.; Yao, X.S. New lignans from the bioactive fraction of Sambucus williamsii Hance and proliferation activities on osteoblastic-like UMR106 cells. Fitoterapia 2010, 94, 29-35. [CrossRef] [PubMed]

19. In, S.J.; Seo, K.H.; Song, N.Y.; Lee, D.S.; Kim, Y.C.; Baek, N.I. Lignans and neolignans from the stems of Vibrunum erosum and their neuroprotective and anti-inflammatory activity. Arch. Pharm. Res. 2015, 338, 26-34. [CrossRef] [PubMed]

20. Uchiyama, T.; Miyase, T.; Ueno, A.; Usmanghani, K. Terpene and lignan glycosides from Pluchea indica. Phytochemistry 1991, 30, 655-657. [CrossRef]

21. Iizuka, M.; Warashina, T.; Noro, T. Bufadienolides and a new lignan from the bulbs of Urginea maritima. Chem. Pharm. Bull. 2001, 49, 282-286. [CrossRef] [PubMed]

22. Sakurai, N.; Nagashima, S.; Kawai, K.; Inoue, T. A new lignan, (-)-berchemol, from Berchemia racemosa. Chem. Pharm. Bull. 1989, 37, 3311-3315. [CrossRef]

23. Ando, H.; Hirai, Y.; Fujii, M.; Hori, Y.; Fukumura, M.; Niiho, Y.; Nakajima, Y.; Shibata, T.; Toriizuka, K.; Ida, Y. The chemical constituents of fresh Gentian root. J. Nat. Med. 2007, 61, 269-279. [CrossRef]

24. Schumacher, B.; Scholle, S.; Hoelzl, J.; Khudeir, N.; Hess, S.; Mueller, C.E. Lignans isolated from Valerian: Identification and characterization of a new olivil derivative with partial agonistic activity at $\mathrm{A}_{1}$ adenosine receptors. J. Nat. Prod. 2002, 65, 1479-1485. [CrossRef] [PubMed]

25. Ngan, N.T.; Quang, T.H.; Tai, B.H.; Song, S.B.; Lee, D.; Kim, Y.H. Anti-inflammatory and PPAR transactivational effects of components from the stem bark of Ginkgo biloba. J. Agric. Food Chem. 2012, 60, 2815-2824. [CrossRef] [PubMed]

26. Baba, H.; Yaoita, Y.; Kikuchi, M. Constituents of the roots of Ligularia dentata Hara. J. Nat. Med. 2007, 61, 472-473. [CrossRef]

27. Pamingle, H.; Snowden, R.L.; Schulte-Elte, K.H. Stereoselective conversion of campholene- to necrodane-type monoterpenes. Novel access to $(-)-(R, R)$ - and $(R, S)$ - $\alpha$-necrodol and the enantiomeric $\gamma$-necrodols. Helv. Chim. Acta 1991, 74, 543-548. [CrossRef]

28. Lei, X.; Sui, P.H. Effect of the ginsenoside Rb1 on the spontaneous contraction of intestinal smooth muscle in mice. World J. Gastroenterol. 2012, 18, 5462-5469.

Sample Availability: Samples of all the compounds are available from the authors.

(C) 2017 by the authors. Licensee MDPI, Basel, Switzerland. This article is an open access article distributed under the terms and conditions of the Creative Commons Attribution (CC BY) license (http:/ / creativecommons.org/licenses/by/4.0/). 